\title{
On the Dedekind completion of function rings
}

\author{
Imanol Mozo Carollo, Javier Gutiérrez García and Jorge Picado
}

\begin{abstract}
This paper introduces the frame of partially defined real numbers and the latticeordered ring of partial real functions on a frame. This is then used to construct the order completion of rings of pointfree continuous real functions. The bounded and integervalued cases are also analysed. The application of this pointfree approach to the classical case $\mathrm{C}(X)$ of the ring of continuous real-valued functions on a topological space $X$ yields a new construction for the Dedekind completion of $\mathrm{C}(X)$, considerably more direct and natural than the known procedure using Hausdorff continuous functions.
\end{abstract}

Keywords. Frame of reals, frame of partial reals, continuous real function, partial real function, order complete, Dedekind completion, completely regular frame, extremally disconnected frame, zero-dimensional frame.

2010 Mathematics Subject Classification. 06D22, 06B23, 54C30, 26 A15.

\section{Introduction}

Our main goal with this paper is to construct the Dedekind completion of the ring $\mathrm{C}(X)$ in the most direct and transparent way, avoiding the use of Hausdorff continuous functions in [1]. For that, we approach the problem from a pointfree viewpoint, replacing spaces by an abstraction of their lattices of open sets. The lattices involved here are the frames (or locales), which form the object of study of pointfree topology.

Let $\mathfrak{L}(\mathbb{R})$ denote the frame of reals [3], that is, the frame generated by all ordered pairs $(p, q)$ of rationals, subject to the relations

$$
(p, q) \wedge(r, s)=(p \vee r, q \wedge s)
$$

(R2) $(p, q) \vee(r, s)=(p, s)$ whenever $p \leq r<q \leq s$,

Research supported by the Ministry of Economy and Competitiveness of Spain (under grant MTM2012-37894-C02-02), the UPV/EHU (under grants UFI11/52 and GIU12/39), and the Centre for Mathematics of the University of Coimbra (funded by the European Regional Development Fund through the program COMPETE and by the Portuguese Government through the Fundação para a Ciência e a Tecnologia, under the project PEst-C/MAT/UI0324/2011). 
(R3) $(p, q)=\bigvee\{(r, s) \mid p<r<s<q\}$,

(R4) $\bigvee\{(p, q) \mid p, q \in \mathbb{Q}\}=1$.

For any frame $L$ the real continuous functions on $L$ are the frame homomorphisms $\mathfrak{L}(\mathbb{R}) \rightarrow L$. They form a lattice-ordered ring ( $\ell$-ring) [3] that we shall denote by $\mathrm{C}(L)$. The correspondence $L \mapsto \mathrm{C}(L)$ extends that for spaces: if $L=\mathcal{O} X$ (the frame of open sets of a space $X$ ), then the classical function ring $\mathrm{C}(X)$ is naturally isomorphic to $\mathrm{C}(L)$, see [3].

Order completeness of an $\ell$-ring [7], it may be recalled, means that each nonvoid set of elements that is bounded from above has a supremum. What can one say about the Dedekind completion of $\mathrm{C}(L)$ and, in particular, of $\mathrm{C}(X)$ for any space $X$ ? In general, due to axiom (R2) above, $\mathrm{C}(L)$ fails to be order complete. The best known result is a theorem of Banaschewski and Hong [7] that extends familiar facts concerning topological spaces that go back to Nakano [18] and Stone [23]: for a completely regular $L$, the ring $\mathrm{C}(L)$ is order complete if and only if $L$ is extremally disconnected if and only if $L$ is zero-dimensional and the Boolean part of $L$ is complete.

That the completion of $\mathrm{C}(L)$ exists at all is a classical theorem that traces back to Dedekind and was fully articulated by MacNeille [17] (see $[8,10]$ for details). What is sought here is a pointfree construction of the order completion of $\mathrm{C}(L)$ in the category of function rings. In order to achieve it we must find in some way the smallest order complete lattice containing $\mathrm{C}(L)$. A natural idea is to avoid the problem caused by (R2) by deleting it from the list of axioms. So our main device will be the frame $\mathfrak{L}(\mathbb{R})$ of partially defined real numbers, presented by the same generators as $\mathfrak{L}(\mathbb{R})$ and by all relations except relation (R2). Of course, this is a bigger frame in which $\mathfrak{L}(\mathbb{R})$ embeds canonically. Then $\mathrm{C}(L)$ also embeds canonically in the class $\operatorname{IC}(L)=\operatorname{Frm}(\mathfrak{L}(\mathbb{R}), L)$ of partial real functions on $L$. We prove that $\operatorname{IC}(L)$ is Dedekind complete (Section 2) and describe (in Section 3) the Dedekind completion $D(\mathrm{C}(L))$ of $\mathrm{C}(L)$ inside $\mathrm{IC}(L)$ by

$$
\begin{gathered}
D(\mathrm{C}(L))=\{h \in \mathrm{IC}(L) \mid \text { (a) there exist } f, g \in \mathrm{C}(L) \text { such that } f \leq h \leq g, \\
\text { (b) } h(p,-)^{*} \leq h(-, q) \text { and } h(-, q)^{*} \leq h(p,-) \\
\text { for any } p<q\} .
\end{gathered}
$$

We also show that, alternatively, the elements of $D(\mathrm{C}(L))$ are precisely the maximal elements of $\operatorname{IC}(L)$ (with respect to a certain partial order $\sqsubseteq$ ) that satisfy condition (a). The bounded and integer-valued cases are then analysed (Sections 4 and 5). In particular, the aforementioned result of Banaschewski-Hong [7] follows as an immediate corollary from our construction. Finally, in Section 6, we show that the application of these ideas to the classical case of the ring $\mathrm{C}(X)$ of continuous 
real-valued functions on a topological space $X$ provides a new construction for its order completion. In particular, the results of Anguelov [1] and Danet [11] follow easily from our approach.

\section{Preliminaries}

\subsection{Order completion}

For any subset $A$ of a partially ordered set $(P, \leq)$, we will denote by $\bigvee^{P} A$ (resp. $\bigwedge^{P} A$ ) the supremum (resp. infimum) of $A$ in $P$ in case it exists (we shall omit the superscript if it is clear from the context).

A Dedekind-MacNeille completion (also called normal completion, completion by cuts or just MacNeille completion) of a poset $P$ is a join- and meet-dense embedding $\varphi: P \rightarrow M(P)$ in a complete lattice (as usual, a map $\varphi: P \rightarrow M(P)$ is said to be join-dense if and only if so is its image in $M(P)$; that is, each element of $M(P)$ is a join of elements from $\varphi[P]$; meet-density is defined dually). The Dedekind-MacNeille completion is the only complete lattice in which the given poset is join- and meet-dense.

There are various abstract characterizations of Dedekind-MacNeille completions (see, e.g. $[5,8,9,20,22]$ ). The existence of such completions is given by the well-known construction of MacNeille [8, 17].

For our purposes in this paper, since we are dealing with a $P$ that is a group, it will be more useful to consider a completion of $P$, denoted by $D(P)$, that is slightly smaller than $M(P)$ in case $P$ has no 0 and 1 [22, Theorem 1.3.4]. It is called the Dedekind completion of $P$ and it is defined in the following way $[7,10,22]$ :

A poset $(P, \leq)$ is Dedekind complete (elsewhere also referred as conditionally complete) if every non-void subset $A$ of $P$ which is bounded from above has a supremum in $P$ (in particular, every non-void subset $B$ of $P$ which is bounded from below will have a infimum in $P$ ). A Dedekind completion of a poset $P$ is a join- and meet-dense embedding $\Phi: P \rightarrow D(P)$ in a Dedekind complete poset. $D(P)$ is the only Dedekind complete poset in which the given poset is join- and meet-dense. So $D(P)$ satisfies

$$
\hat{p}=\bigvee^{D(P)}\{\Phi(p) \mid \Phi(p) \leq \hat{p}\}=\bigwedge^{D(P)}\{\Phi(p) \mid \Phi(p) \geq \hat{p}\}
$$

for every $\hat{p} \in D(P)$.

For more information on universal properties of the Dedekind completion see $[22,1.3]$. 


\subsection{Frames}

A frame (or locale) $L$ is a complete lattice such that $a \wedge \bigvee B=\bigvee\{a \wedge b \mid b \in B\}$ for all $a \in L$ and $B \subseteq L$; equivalently, it is a complete Heyting algebra with Heyting operation $\rightarrow$ satisfying the standard equivalence $a \wedge b \leq c$ if and only if $a \leq b \rightarrow c$. The pseudocomplement of an $a \in L$ is the element

$$
a^{*}=a \rightarrow 0=\bigvee\{b \in L \mid a \wedge b=0\}
$$

An element $a$ is regular if $a^{* *}=a$ (equivalently, if $a=b^{*}$ for some $b$ ). A frame homomorphism is a map $h: L \rightarrow M$ between frames which preserve finitary meets (including the top element 1) and arbitrary joins (including the bottom element 0 ). Then Frm is the corresponding category of frames and their homomorphisms.

The most typical example of a frame is the lattice $\mathcal{O} X$ of open subsets of a topological space $X$. The correspondence $X \mapsto \mathcal{O} X$ is clearly functorial (by taking inverse images), and consequently we have a contravariant functor $\mathcal{O}:$ Top $\rightarrow$ Frm where Top denotes the category of topological spaces and continuous maps. There is also a functor in the opposite direction, the spectrum functor $\Sigma$ : Frm $\rightarrow$ Top which assigns to each frame $L$ its spectrum $\Sigma L$, the space of all homomorphisms $\xi: L \rightarrow\{0,1\}$ with open sets $\Sigma_{a}=\{\xi \in \Sigma L \mid \xi(a)=1\}$ for any $a \in L$, and to each frame homomorphism $h: L \rightarrow M$ the continuous map $\Sigma h: \Sigma M \rightarrow \Sigma L$ such that $\Sigma h(\xi)=\xi h$. The spectrum functor is right adjoint to $\mathcal{O}$, with adjunction maps $\eta_{L}: L \rightarrow \mathcal{O} \Sigma L, \eta_{L}(a)=\Sigma_{a}$ and $\epsilon_{X}: X \rightarrow \Sigma \mathcal{O} X, \epsilon_{X}(x)=\hat{x}, \hat{x}(U)=1$ if and only if $x \in U$ (the former is the spatial reflection of the frame $L$ ).

For general notions and results concerning frames we refer to Johnstone [14] or the recent Picado-Pultr [19]. The particular notions we will need are the following: a frame $L$ is

- completely regular if $a=\bigvee\{b \in L \mid b \prec \prec a\}$ for each $a \in L$, where $b \prec \prec a$ means that there is $\left\{c_{r} \mid r \in \mathbb{Q} \cap[0,1]\right\} \subseteq L$ such that $a \leq c_{0}, c_{1} \leq b$ and $c_{r} \prec c_{s}$ (i.e. $c_{r}^{*} \vee c_{s}=1$ ) whenever $r<s$;

- extremally disconnected if $a^{*} \vee a^{* *}=1$ for every $a \in L$; and

- zero-dimensional if each element of $L$ is a join of complemented elements.

\subsection{Real functions}

It will be useful here (as it has been also in [6]) to adopt the equivalent description of $\mathfrak{L}(\mathbb{R})$ with the elements $(r,-)=\bigvee_{s \in \mathbb{Q}}(r, s)$ and $(-, s)=\bigvee_{r \in \mathbb{Q}}(r, s)$ as primitive notions. 
Specifically, the frame of reals $\mathfrak{L}(\mathbb{R})$ is equivalently defined by generators $(r,-)$ and $(-, r)$ for $r \in \mathbb{Q}$ and the following relations:

(r1) $(r,-) \wedge(-, s)=0$ whenever $r \geq s$,

(r2) $(r,-) \vee(-, s)=1$ whenever $r<s$,

(r3) $(r,-)=\bigvee_{s>r}(s,-)$, for every $r \in \mathbb{Q}$,

(r4) $(-, r)=\bigvee_{s<r}(-, s)$, for every $r \in \mathbb{Q}$,

(r5) $\bigvee_{r \in \mathbb{Q}}(r,-)=1$,

(r6) $\bigvee_{r \in \mathbb{Q}}(-, r)=1$.

With $(p, q)=(p,-) \wedge(-, q)$ one goes back to (R1)-(R4).

Regarding the frame homomorphisms $\mathfrak{L}(\mathbb{R}) \rightarrow L$, for a general frame $L$, as the continuous real functions on $L$ provides a natural extension of the classical notion since continuous real functions on a space $X$ may be represented as frame homomorphisms $h: \mathfrak{L}(\mathbb{R}) \rightarrow \mathcal{O} X$ (see [3] for a detailed account).

There is a useful way of specifying continuous real functions on $L$ with the help of scales. A scale in $L$ is a map $\sigma: \mathbb{Q} \rightarrow L$ such that

(1) $\sigma(r) \vee \sigma(s)^{*}=1$ whenever $r<s$,

(2) $\bigvee_{r \in \mathbb{Q}} \sigma(r)=1=\bigvee_{r \in \mathbb{Q}} \sigma(r)^{*}$.

For any scale $\sigma$ the formulas

$$
f(r,-)=\bigvee\{\sigma(s) \mid s>r\} \quad \text { and } \quad f(-, r)=\bigvee\left\{\sigma(s)^{*} \mid s<r\right\} \quad(r \in \mathbb{Q})
$$

determine a continuous real function $f: \mathfrak{L}(\mathbb{R}) \rightarrow L$.

Note. The meaning of the term scale used here differs from its use in [14] where it refers to maps from the unit interval of $\mathbb{Q}$ (and not all of $\mathbb{Q}$ ) into $L$. In [3] the term descending trail is used instead.

The ring $\mathrm{C}(L)=\operatorname{Frm}(\mathfrak{L}(\mathbb{R}), L)$ is partially ordered by

$$
\begin{array}{rlll}
f \leq g & \text { iff } \quad & f(r,-) \leq g(r,-) & \text { for all } r \in \mathbb{Q} \\
& \text { iff } \quad g(-, r) \leq f(-, r) & \text { for all } r \in \mathbb{Q} .
\end{array}
$$

Example 1.1. (1) For each $r \in \mathbb{Q}$, the constant function $r$ determined by $r$ is defined by

$$
\boldsymbol{r}(s,-)=\left\{\begin{array}{ll}
0 & \text { if } s \geq r, \\
1 & \text { if } s<r,
\end{array} \quad \text { and } \quad \boldsymbol{r}(-, s)= \begin{cases}1 & \text { if } s>r \\
0 & \text { if } s \leq r\end{cases}\right.
$$

for every $s \in \mathbb{Q}$. 
(2) For each complemented $a \in L$, the characteristic function $\chi_{a}$ determined by $a$ is given by

$$
\chi_{a}(s,-)=\left\{\begin{array}{ll}
0 & \text { if } s \geq 1, \\
a & \text { if } 0 \leq s<1, \\
1 & \text { if } s<0,
\end{array} \quad \text { and } \quad \chi_{a}(-, s)= \begin{cases}1 & \text { if } s>1, \\
a * & \text { if } 0<s \leq 1 \\
0 & \text { if } s \leq 0\end{cases}\right.
$$

for every $s \in \mathbb{Q}$.

An $f \in \mathrm{C}(L)$ is said to be bounded if there exist $p, q \in \mathbb{Q}$ such that $\boldsymbol{p} \leq f \leq \boldsymbol{q}$. Equivalently, $f$ is said to be bounded if and only if there is some rational $r$ such that $f((-,-r) \vee(r,-))=0$, that is, $f(-r, r)=1$. We shall denote by $\mathrm{C}^{*}(L)$ the set of all bounded members of $\mathrm{C}(L)$. Obviously, all constant functions and all characteristic functions are in $\mathrm{C}^{*}(L)$.

As it is well known, in general neither $\mathrm{C}(L)$ nor $\mathrm{C}^{*}(L)$ are Dedekind complete [7].

The operations on the algebra $\mathrm{C}(L)$ are determined by the operations of $\mathbb{Q}$ as lattice-ordered ring as follows (see [3] and [13] for more details):

(1) For $\diamond=+, \cdot \wedge, \vee$,

$$
(f \diamond g)(p, q)=\bigvee\{f(r, s) \wedge g(t, u) \mid\langle r, s\rangle \diamond\langle t, u\rangle \subseteq\langle p, q\rangle\}
$$

where $\langle\cdot, \cdot\rangle$ stands for open interval in $\mathbb{Q}$ and the inclusion on the right means that $x \diamond y \in\langle p, q\rangle$ whenever $x \in\langle r, s\rangle$ and $y \in\langle t, u\rangle$.

(2) $(-f)(p, q)=f(-q,-p)$.

(3) For each $r \in \mathbb{Q}$, the nullary operation $\boldsymbol{r}$ is defined as in Example 1.1 (1) above.

(4) For each $0<\lambda \in \mathbb{Q},(\lambda \cdot f)(p, q)=f\left(\frac{p}{\lambda}, \frac{q}{\lambda}\right)$.

These operations satisfy all the identities which hold for their counterparts in $\mathbb{Q}$ and hence they determine an $f$-ring structure in $\mathrm{C}(L)$.

\section{Partial real functions}

Let $\mathbb{R}$ denote the set of compact intervals $\boldsymbol{a}=[\underline{a}, \bar{a}]$ of the real line ordered by reverse inclusion (which we denote by $\sqsubseteq$ ):

$$
\boldsymbol{a} \sqsubseteq \boldsymbol{b} \quad \text { iff } \quad[\underline{a}, \bar{a}] \supseteq[\underline{b}, \bar{b}] \quad \text { iff } \quad \underline{a} \leq \underline{b} \leq \bar{b} \leq \bar{a} .
$$

The pair $(\mathbb{I R}, \sqsubseteq)$ is a domain [12], referred to as the partial real line (also interval-domain). The interval domain was proposed by Dana Scott in [21] as a 
domain-theoretic model for the real numbers. It is a successful idea that has inspired a number of computational models for real numbers.

The way-below relation of $\mathbb{I}$ is given by

$$
\boldsymbol{a} \ll \boldsymbol{b} \quad \text { iff } \quad \underline{a}<\underline{b} \leq \bar{b}<\bar{a}
$$

and we denote

$$
\uparrow \boldsymbol{a}=\{\boldsymbol{b} \in \mathbb{I} \mathbb{R} \mid \boldsymbol{a} \ll \boldsymbol{b}\} .
$$

The family $\{\uparrow \boldsymbol{a} \mid \boldsymbol{a} \in \mathbb{I} \mathbb{R}, \underline{a}, \bar{a} \in \mathbb{Q}\}$ forms a countable basis of the Scott topology $\mathcal{O} \mathbb{R}$ on $(\mathbb{I R}, \sqsubseteq)$.

Remark 2.1. (1) Let $\pi_{1}, \pi_{2}: \mathbb{I} \mathbb{R} \rightarrow \mathbb{R}$ be the projections defined for each $\boldsymbol{a} \in \mathbb{I} \mathbb{R}$ by $\pi_{1}(\boldsymbol{a})=\underline{a}$ and $\pi_{2}(\boldsymbol{a})=\bar{a}$. Then for each $r \in \mathbb{Q}$,

$$
\begin{aligned}
\pi_{1}^{-1}(r,+\infty) & =\{\boldsymbol{a} \in \mathbb{I} \mathbb{R} \mid r<\underline{a}\} \\
& =\bigcup_{\beta \in \mathbb{R}, \beta>r}\{\boldsymbol{a} \in \mathbb{I} \mathbb{R} \mid r<\underline{a} \leq \bar{a}<\beta\} \\
& =\bigcup_{\beta \in \mathbb{R}, \beta>r} \uparrow[r, \beta]
\end{aligned}
$$

and

$$
\begin{aligned}
\pi_{2}^{-1}(-\infty, r) & =\{\boldsymbol{a} \in \mathbb{I} \mathbb{R} \mid \bar{a}<r\} \\
& =\bigcup_{\alpha \in \mathbb{R}, \alpha<r}\{\boldsymbol{a} \in \mathbb{I} \mathbb{R} \mid \alpha<\underline{a} \leq \bar{a}<r\} \\
& =\bigcup_{\alpha \in \mathbb{R}, \alpha<r} \uparrow[\alpha, r] .
\end{aligned}
$$

It follows that for the upper $\tau_{u}$ and lower $\tau_{l}$ topologies in $\mathbb{R}, \pi_{1}: \mathbb{I} \rightarrow\left(\mathbb{R}, \tau_{u}\right)$ is continuous, (i.e., $\pi_{1}$ is lower semicontinuous) and $\pi_{2}: \mathbb{R} \rightarrow\left(\mathbb{R}, \tau_{l}\right)$ is continuous, (i.e., $\pi_{2}$ is upper semicontinuous). Hence, for any $f \in \mathrm{C}(X, \mathbb{R})$, we have $\pi_{1} \circ f \in \operatorname{LSC}(X, \mathbb{R}), \pi_{2} \circ f \in \operatorname{USC}(X, \mathbb{R})$ and $\pi_{1} \circ f \leq \pi_{2} \circ f$.

Note further that for each $\boldsymbol{a} \in \mathbb{I} \mathbb{R}$ one has $\uparrow \boldsymbol{a}=\pi_{1}^{-1}(\underline{a},+\infty) \cap \pi_{2}^{-1}(-\infty, \bar{a})$. Consequently, the Scott topology on $\mathbb{I} \mathbb{R}$ is the initial topology with respect to $\pi_{1}: \mathbb{I} \rightarrow\left(\mathbb{R}, \tau_{u}\right)$ and $\pi_{2}: \mathbb{I} \rightarrow\left(\mathbb{R}, \tau_{l}\right)$.

(2) Let $e: \mathbb{R} \rightarrow \mathbb{R}$ be given by $e(a)=[a, a]$ for each $a \in \mathbb{R}$. It is easy to check that $e$ is an embedding of $\mathbb{R}$ endowed with the usual topology into $(\mathbb{R}, \mathcal{O} \mathbb{R})$. Sometimes we shall identify $\mathbb{R}$ with its homeomorphic copy $e(\mathbb{R}) \subseteq \mathbb{I}$. Similarly, a real-valued function $f: X \rightarrow \mathbb{R}$ will be identified with $e \circ f: X \rightarrow \mathbb{I}$. 
When investigating the existence of suprema of families of continuous real functions on a frame, one immediately realizes that the problem lies on the defining relation $(\mathrm{r} 2)$ ( or $(\mathrm{R} 2)$ ). This urged us to consider the partial variant of $\mathfrak{L}(\mathbb{R})$ defined by generators $(r,-)$ and $(-, r)$ for $r \in \mathbb{Q}$ and relations (r1), (r3)-(r6). We call it the frame of partial reals $\mathfrak{L}(\mathbb{R})$. There is of course a basic homomorphism $\varrho: \mathfrak{L}(\mathbb{I R}) \rightarrow \mathfrak{L}(\mathbb{R})$ defined on generators by $(r,-) \mapsto(r,-)$ and $(-, r) \mapsto(-, r)$.

Proposition 2.2. The space of partial reals with the Scott topology is homeomorphic to $\Sigma \mathfrak{L}(\mathbb{I R})$. The homeomorphism $\tau: \Sigma \mathfrak{L}(\mathbb{I}) \rightarrow \mathbb{I} \mathbb{R}$ is such that

$$
\underline{\tau(\xi)}=\bigvee\{r \in \mathbb{Q} \mid \xi(r,-)=1\} \quad \text { and } \quad \overline{\tau(\xi)}=\bigwedge\{s \in \mathbb{Q} \mid \xi(-, s)=1\}
$$

for each $\xi \in \Sigma \mathfrak{L}(\mathbb{R})$.

Proof. Let $\xi \in \Sigma \mathfrak{L}(\mathbb{I R})$. We first note that by (r1), (r5) and (r6) there exists a pair of rationals $r_{1}<r_{2}$ such that

$$
\xi\left(-, r_{1}\right)=\xi\left(r_{2},-\right)=0 \quad \text { and } \quad \xi\left(r_{1},-\right)=\xi\left(-, r_{2}\right)=1 .
$$

Indeed, if $\xi(r,-)=0$ for every $r \in \mathbb{Q}$, then $\xi\left(\bigvee_{r \in \mathbb{Q}}(r,-)\right)=\bigvee_{r \in \mathbb{Q}} \xi(r,-)=0$, contradicting (r5) by the compactness of $\{0,1\}$. Therefore there exists some $r_{1} \in \mathbb{Q}$ such that $\xi\left(r_{1},-\right)=1$ and then, by $(\mathrm{r} 1)$,

$$
0=\xi(0)=\xi\left(\left(r_{1},-\right) \wedge\left(-, r_{1}\right)\right)=\xi\left(-, r_{1}\right) .
$$

By a similar argument, using (r1) and (r6), we may conclude that $\xi\left(-, r_{2}\right)=1$ and $\xi\left(r_{2},-\right)=1$ for some $r_{2} \in \mathbb{Q}$. Finally,

$$
1=\xi\left(r_{1},-\right) \wedge \xi\left(-, r_{2}\right)=\xi\left(\left(r_{1},-\right) \wedge\left(-, r_{2}\right)\right)
$$

implies $r_{1}<r_{2}$, by (r1).

It now follows that we have

$$
\underline{\tau(\xi)}=\bigvee\{r \in \mathbb{Q} \mid \xi(r,-)=1\} \in \mathbb{R}
$$

and

$$
\overline{\tau(\xi)}=\bigwedge\{s \in \mathbb{Q} \mid \xi(-, s)=1\} \in \mathbb{R} .
$$

For any such $r, s$,

$$
\xi((r,-) \wedge(-, s))=\xi(r,-) \wedge \xi(-, s)=1
$$

and thus, by (r1), $r<s$. Hence $\underline{\tau(\xi)} \leq \overline{\tau(\xi)}$ and $\tau(\xi)=[\underline{\tau(\xi)}, \overline{\tau(\xi)}]$ belongs in fact to $\mathbb{I R}$. 
In order to show that $\tau$ is one-to-one, let $\xi_{1} \neq \xi_{2}$. Then there exists an $r \in \mathbb{Q}$ such that, say, $\xi_{1}(r,-)=1$ and $\xi_{2}(r,-)=0$. Then, by (r3),

$$
1=\xi_{1}(r,-)=\xi_{1}\left(\bigvee_{p>r}(p,-)\right) \text {. }
$$

Thus there exists a $p>r$ such that $\xi_{1}(p,-)=1$, and hence $r<p \leq \tau\left(\xi_{1}\right)$. On the other hand, since $\xi_{2}(q,-)=0$ for each $q \geq r$, it follows that

$$
\underline{\tau\left(\xi_{2}\right)}=\bigvee\left\{q \in \mathbb{Q} \mid \xi_{2}(q,-)=1\right\} \leq r .
$$

Hence $\tau\left(\xi_{2}\right) \leq r<p \leq \tau\left(\xi_{1}\right)$. The arguments for the other cases are analogous.

The function $\tau$ is also surjective. Indeed, given $\boldsymbol{a} \in \mathbb{I R}$, let $\xi_{\boldsymbol{a}}: \mathfrak{L}(\mathbb{I} \mathbb{R}) \rightarrow\{0,1\}$ be given by $\xi_{\boldsymbol{a}}(r,-)=1$ if and only if $r<\underline{a}$ and $\xi_{\boldsymbol{a}}(-, r)=1$ if and only if $\bar{a}<r$ for every $r \in \mathbb{Q}$. It is easy to check that this correspondence turns the defining relations (r1), (r3)-(r6) into identities in $\{0,1\}$ and so each $\xi_{\boldsymbol{a}}$ is a frame homomorphism. Moreover

$$
\underline{\tau\left(\xi_{\boldsymbol{a}}\right)}=\bigvee\left\{r \in \mathbb{Q} \mid \xi_{\boldsymbol{a}}(r,-)=1\right\}=\bigvee\{r \in \mathbb{Q} \mid r<\underline{a}\}=\underline{a}
$$

and

$$
\overline{\tau\left(\xi_{\boldsymbol{a}}\right)}=\bigwedge\left\{r \in \mathbb{Q} \mid \xi_{\boldsymbol{a}}(-, r)=1\right\}=\bigwedge\{r \in \mathbb{Q} \mid \bar{a}<r\}=\bar{a} .
$$

Hence $\tau\left(\xi_{\boldsymbol{a}}\right)=\boldsymbol{a}$.

It remains to show $\tau$ is a homeomorphism. Now, for each basic Scott open set $\uparrow \boldsymbol{a}$ (with $\boldsymbol{a} \in \mathbb{I} \mathbb{R}$ and $\underline{a}, \bar{a} \in \mathbb{Q}$ ) we have that

$$
\begin{aligned}
\tau^{-1}(\uparrow \boldsymbol{a}) & =\left\{\xi_{\boldsymbol{b}} \in \Sigma \mathfrak{L}(\mathbb{I} \mathbb{R}) \mid \boldsymbol{a} \ll \boldsymbol{b}\right\} \\
& =\left\{\xi_{\boldsymbol{b}} \in \Sigma \mathfrak{L}(\mathbb{I} \mathbb{R}) \mid \underline{a}<\underline{b} \leq \bar{b}<\bar{a}\right\} \\
& =\left\{\xi_{\boldsymbol{b}} \in \Sigma \mathfrak{L}(\mathbb{I} \mathbb{R}) \mid \xi_{\boldsymbol{b}}(\underline{a},-)=1 \text { and } \xi_{\boldsymbol{b}}(-, \bar{a})=1\right\} \\
& =\Sigma_{(\underline{a},-)} \cap \Sigma_{(-, \bar{a})} .
\end{aligned}
$$

Hence $\tau$ is continuous. On the other hand, for any open sets $\Sigma_{(r,-)}$ or $\Sigma_{(-, r)}$ of $\Sigma \mathfrak{L}(\mathbb{I} \mathbb{R})$,

$$
\begin{aligned}
\tau\left(\Sigma_{(r,-)}\right) & =\{\tau(\xi) \mid \xi \in \Sigma \mathfrak{L}(\mathbb{I} \mathbb{R}) \text { and } \xi(r,-)=1\} \\
& =\left\{\tau\left(\xi_{\boldsymbol{a}}\right) \mid \boldsymbol{a} \in \mathbb{I} \mathbb{R} \text { and } \xi_{\boldsymbol{a}}(r,-)=1\right\} \\
& =\{\boldsymbol{a} \in \mathbb{I} \mathbb{R} \mid r<\underline{a}\} \\
& =\bigcup_{\beta \in \mathbb{R}, \beta>r}\{\boldsymbol{a} \in \mathbb{I} \mathbb{R} \mid r<\underline{a} \leq \bar{a}<\beta\}=\bigcup_{\beta \in \mathbb{R}, \beta>r} \uparrow[r, \beta] .
\end{aligned}
$$


and

$$
\begin{aligned}
\tau\left(\Sigma_{(-, r)}\right) & =\{\tau(\xi) \mid \xi \in \Sigma \mathfrak{L}(\mathbb{I R}) \text { and } \xi(-, r)=1\} \\
& =\left\{\tau\left(\xi_{\boldsymbol{a}}\right) \mid \boldsymbol{a} \in \mathbb{I} \mathbb{R} \text { and } \xi_{\boldsymbol{a}}(-, r)=1\right\} \\
& =\{\boldsymbol{a} \in \mathbb{R} \mid \overline{\mathbb{a}}<r\} \\
& =\bigcup_{\alpha \in \mathbb{R}, \alpha<r}\{\boldsymbol{a} \in \mathbb{R} \mid \alpha<\underline{a} \leq \bar{a}<r\}=\bigcup_{\alpha \in \mathbb{R}, \alpha<r} \uparrow[\alpha, r]
\end{aligned}
$$

are Scott open sets.

Remark 2.3. The homeomorphism $\tau^{-1}: \mathbb{I} \mathbb{R} \rightarrow \Sigma \mathfrak{L}(\mathbb{R})$ induces an isomorphism

$$
\mathcal{O} \Sigma \mathfrak{L}(\mathbb{I R}) \rightarrow \mathcal{O} \mathbb{R}, \quad \Sigma_{(r,-)} \mapsto \pi_{1}^{-1}(r,+\infty), \quad \Sigma_{(-, r)} \mapsto \pi_{2}^{-1}(-\infty, r) .
$$

Thus the homomorphism $\mathfrak{L}(\mathbb{I R}) \rightarrow \mathcal{O} \mathbb{R}$ taking $(r,-)$ to $\pi_{1}^{-1}(r,+\infty)$ and $(-, r)$ to $\pi_{2}^{-1}(-\infty, r)$ is the spatial reflection map $\eta_{\mathfrak{L}(\mathbb{R})}$ of the frame of partial real numbers. Note that this homomorphism is an isomorphism. Indeed, $\eta_{\mathfrak{L}(\mathbb{R})}$ is onto, since for each $\boldsymbol{a} \in \mathbb{I} \mathbb{R}$ with $\underline{a}, \bar{a} \in \mathbb{Q}$,

$$
\eta_{\mathfrak{L}(\mathbb{R} \mathbb{R})}((\underline{a},-) \wedge(-, \bar{a}))=\{\boldsymbol{b} \in \mathbb{I} \mathbb{R} \mid \underline{a}<\underline{b} \text { and } \bar{b}<\bar{a}\}=\uparrow \boldsymbol{a},
$$

and $\Psi: \mathcal{O} \mathbb{R} \rightarrow \mathfrak{L}(\mathbb{I})$, given by

$$
\Psi(\uparrow \boldsymbol{a})=(\underline{a},-) \wedge(-, \bar{a}) \quad \text { for each } \boldsymbol{a} \in \mathbb{I} \mathbb{R} \text { such that } \underline{a}, \bar{a} \in \mathbb{Q},
$$

is a left inverse of $\eta_{\mathfrak{L}(\mathbb{R})}$ :

$$
\begin{aligned}
\Psi \circ \eta_{\mathfrak{L}(\mathbb{R})}(r,-) & =\Psi(\{\boldsymbol{a} \in \mathbb{I} \mathbb{R} \mid r<\underline{a}\}) \\
& =\Psi(\bigcup\{\uparrow \boldsymbol{b} \mid \underline{b}=r, \bar{b} \in \mathbb{Q}\}) \\
& =\bigvee\{(r,-) \wedge(-, \bar{b}) \mid \bar{b} \in \mathbb{Q}\} \\
& =(r,-) \wedge \bigvee\{(-, \bar{b}) \mid \bar{b} \in \mathbb{Q}\}=(r,-),
\end{aligned}
$$

and

$$
\begin{aligned}
\Psi \circ \eta_{\mathfrak{L}(\mathbb{R})}(-, r) & =\Psi(\{\boldsymbol{a} \in \mathbb{I} \mathbb{R} \mid \bar{a}<r\}) \\
& =\Psi(\bigcup\{\uparrow \boldsymbol{b} \mid \underline{b} \in \mathbb{Q}, \bar{b}<r\}) \\
& =\bigvee\{(\underline{b},-) \wedge(-, r) \mid \underline{b} \in \mathbb{Q}\} \\
& =(-, r) \wedge \bigvee\{(\underline{b},-) \mid \bar{b} \in \mathbb{Q}\}=(-, r) .
\end{aligned}
$$


Definition 2.4. A continuous partial real function on a frame $L$ is a frame homomorphism $h: \mathfrak{L}(\mathbb{I} \mathbb{R}) \rightarrow L$.

As in the case of continuous real functions on a space $X$, one can easily show that continuous functions $X \rightarrow \mathbb{I}$ may be represented as frame homomorphisms $h: \mathfrak{L}(\mathbb{R}) \rightarrow \mathcal{O} X$, which justifies the preceding definition:

Corollary 2.5. For each topological space $(X, \mathcal{O} X)$ there is a natural isomorphism

$$
\Phi: \operatorname{Frm}(\mathfrak{L}(\mathbb{I} \mathbb{R}), \mathcal{O} X) \stackrel{\sim}{\rightarrow} \operatorname{Top}(X, \mathbb{I} \mathbb{R}) .
$$

Proof. By the (dual) adjunction between the contravariant functors $\mathcal{O}:$ Top $\rightarrow$ Frm and $\Sigma:$ Frm $\rightarrow$ Top there is a natural isomorphism $\operatorname{Frm}(L, \mathcal{O} X) \stackrel{\sim}{\rightarrow} \operatorname{Top}(X, \Sigma L)$ for all $L$ and $X$. Combining this for the case $L=\mathfrak{L}(\mathbb{I R})$ with the homeomorphism $\tau: \Sigma(\mathfrak{L}(\mathbb{I} \mathbb{R})) \rightarrow \mathbb{I} \mathbb{R}$ from Proposition 2.2 one obtains the isomorphism.

Specifically, $\Phi$ is given by the correspondence $h \mapsto \tilde{h}$ where $\tilde{h}(x)=[\bigvee\{r \in \mathbb{Q} \mid x \in h(r,-)\}, \bigwedge\{r \in \mathbb{Q} \mid x \in h(-, r)\}]$ for every $x \in X$ In the opposite direction, given $f \in \mathrm{C}(X, \mathbb{I} \mathbb{R})$ the corresponding $h$ is defined by

$$
\begin{aligned}
& h(r,-)=\left(\pi_{1} \circ f\right)^{-1}(r,+\infty), \\
& h(-, r)=\left(\pi_{2} \circ f\right)^{-1}(-\infty, r) \quad \text { for every } r \in \mathbb{Q} .
\end{aligned}
$$

We shall denote by $\operatorname{IC}(L)$ the set $\operatorname{Frm}(\mathfrak{L}(\mathbb{I R}), L)$, partially ordered by

$$
f \leq g \quad \text { iff } \quad f(r,-) \leq g(r,-) \text { and } g(-, r) \leq f(-, r) \quad \text { for all } r \in \mathbb{Q} .
$$

Remark 2.6. (1) The functions $h \in \mathrm{IC}(L)$ that factor through the canonical insertion $\varrho: \mathfrak{L}(\mathbb{R}) \rightarrow \mathfrak{L}(\mathbb{R})$ are just those which satisfy $h(r,-) \vee h(-, s)=1$ whenever $r<s$. In view of this, we will keep the notation $\mathrm{C}(L)$ to denote also the class inside $\mathrm{IC}(L)$ of the functions $h$ such that $h(r,-) \vee h(-, s)=1$ whenever $r<s$.

(2) In case $f \in \mathrm{C}(L)$, as in (1.3.1), the second condition on $f$ and $g$ in (2.5.1) is needless because it is equivalent to the first one:

$$
\begin{aligned}
g(-, r) & =g\left(\bigvee_{s<r}(-, s)\right)=\bigvee_{s<r} g(-, s) \\
& \leq \bigvee_{s<r} g(s,-)^{*} \leq \bigvee_{s<r} f(s,-)^{*} \leq f(-, r),
\end{aligned}
$$

the last inequality because $f$ being in $\mathrm{C}(L)$ then, by (r2), $f(s,-) \vee f(-, r)=1$. 
A similar argument shows that the first condition follows from the second one whenever $g \in \mathrm{C}(L)$ and so the two conditions are equivalent if both $f, g$ are in $\mathrm{C}(L)$, as in (1.3.1).

(3) There is an order reversing isomorphism $-(\cdot): \operatorname{IC}(L) \rightarrow \operatorname{IC}(L)$ defined by $(-h)(-, r)=h(-r,-) \quad$ and $\quad(-h)(r,-)=h(-,-r) \quad$ for all $r \in \mathbb{Q}$.

When restricted to $\mathrm{C}(L)$ it yields an isomorphism $\mathrm{C}(L) \rightarrow \mathrm{C}(L)$.

A continuous partial real function $h \in \operatorname{IC}(L)$ is said to be bounded if there exist $p, q \in \mathbb{Q}$ such that $\boldsymbol{p} \leq h \leq \boldsymbol{q}$. Equivalently,

$h$ is bounded iff $\exists r \in \mathbb{Q}$ such that $h(-r, r)=1$.

We shall denote by $\operatorname{IC}^{*}(L)$ the set of bounded functions in $\operatorname{IC}(L)$.

Example 2.7. For each $a, b \in L$ such that $a \wedge b=0$ let $\chi_{a, b}$ denote the bounded continuous partial real function given by

$$
\chi_{a, b}(r,-)=\left\{\begin{array}{ll}
0 & \text { if } r \geq 1, \\
a & \text { if } 0 \leq r<1, \\
1 & \text { if } r<0,
\end{array} \quad \text { and } \quad \chi_{a, b}(-, r)= \begin{cases}1 & \text { if } r>1, \\
b & \text { if } 0<r \leq 1, \\
0 & \text { if } r \leq 0,\end{cases}\right.
$$

for each $r \in \mathbb{Q}$. Clearly, $\chi_{a, b} \in \mathrm{C}^{*}(L)$ if and only if $a \vee b=1$, i.e., if and only if $a$ is complemented with complement $b$.

Proposition 2.8. The class $\mathrm{IC}(L)$ is closed under non-void bounded suprema.

Proof. Let $\left\{h_{i}\right\}_{i \in I} \subseteq \mathrm{IC}(L)$ and $h \in \mathrm{IC}(L)$

$$
h_{i} \leq h \quad \text { for all } i \in I .
$$

For each $r, s \in \mathbb{Q}$ we define $h_{\vee}: \mathfrak{L}(\mathbb{I R}) \rightarrow L$ on generators by

$$
h_{\vee}(r,-)=\bigvee_{i \in I} h_{i}(r,-) \quad \text { and } \quad h_{\vee}(-, s)=\bigvee_{q<s} \bigwedge_{i \in I} h_{i}(-, q) .
$$

This is a frame homomorphism since it turns the defining relations (r1) and (r3)(r6) of $\mathfrak{L}(\mathbb{I} \mathbb{R})$ into identities in $L$ :

(r1) whenever $r \geq s$,

$$
\begin{aligned}
h_{\vee}(r,-) \wedge h_{\vee}(-, s) & \leq \bigvee_{i \in I} \bigvee_{q<s} h_{i}(r,-) \wedge h_{i}(-, q) \\
& \leq \bigvee_{i \in I} h_{i}(r,-) \wedge h_{i}(-, s)=0
\end{aligned}
$$


(r3) for each $r \in \mathbb{Q}$,

$$
\bigvee_{s>r} h_{\vee}(s,-)=\bigvee_{i \in I} \bigvee_{s>r} h_{i}(s,-)=\bigvee_{i \in I} h_{i}(r,-)=h_{\vee}(r,-) .
$$

(r4) for each $r \in \mathbb{Q}$,

$$
\bigvee_{s<r} h_{\vee}(-, s)=\bigvee_{s<r} \bigvee_{q<s} \bigwedge_{i \in I} h_{i}(-, q)=\bigvee_{q<r} \bigwedge_{i \in I} h_{i}(-, q)=h_{\vee}(-, r) .
$$

(r5) $\bigvee_{r \in \mathbb{Q}} h_{\vee}(r,-)=\bigvee_{r \in \mathbb{Q}} \bigvee_{i \in I} h_{i}(r,-)=\bigvee_{i \in I} \bigvee_{r \in \mathbb{Q}} h_{i}(r,-)=1$

(r6) $\bigvee_{s \in \mathbb{Q}} h_{\bigvee}(-, s)=\bigvee_{s \in \mathbb{Q}} \bigvee_{q<s} \bigwedge_{i \in I} h_{i}(-, q) \geq \bigvee_{q \in \mathbb{Q}} h(-, q)=1$

Hence $h_{\vee} \in \operatorname{IC}(L)$. In addition, for each $i \in I$ and $r, s \in \mathbb{Q}$,

$$
h_{i}(r,-) \leq h_{\vee}(r,-) \leq h(r,-)
$$

and

$$
h(-, s)=\bigvee_{q<s} h(-, q) \leq h_{\vee}(-, s) \leq \bigvee_{q<s} h_{i}(-, q)=h_{i}(-, s)
$$

and thus $h_{i} \leq h_{\vee} \leq h$ for every $i \in I$. Finally, if $g \in \operatorname{IC}(L)$ is such that $h_{i} \leq g$ for every $i \in I$, then we have, for each $r, s \in \mathbb{Q}$,

$$
g(r,-) \geq \bigvee_{i \in I} h_{i}(r,-)=h_{\vee}(r,-)
$$

and

$$
g(-, s)=\bigvee_{q<s} g(-, q) \leq \bigvee_{q<s} \bigwedge_{i \in I} h_{i}(-, q)=h_{\vee}(-, s)
$$

and so $h_{\vee} \leq g$. Hence $h_{\vee}$ is in fact the supremum of $\left\{h_{i}\right\}_{i \in I}$ in $\operatorname{IC}(L)$.

Corollary 2.9. The class $\mathrm{IC}(L)$ is Dedekind complete.

\section{The Dedekind completion of $\mathrm{C}(L)$}

Since IC $(L)$ is Dedekind complete, it follows that it contains the Dedekind completion of all its subposets, in particular $\mathrm{C}(L)$. Our next task will be to determine the Dedekind completion of $\mathrm{C}(L)$. As a by-product we shall also determine the Dedekind completion of $\mathrm{C}(L)$ in the sense of [7].

We first note that, as explained in [7, Section 2], there is no essential loss of generality if we restrict ourselves to completely regular frames. So, in the sequel, all frames will be taken as completely regular. We start by establishing a couple of lemmas: 
Lemma 3.1. Let $L$ be a completely regular frame and let $h \in \mathrm{IC}(L)$ be such that

(1) $\{f \in \mathrm{C}(L) \mid f \leq h\} \neq \varnothing$,

(2) $h(p,-)^{*} \leq h(-, q)$ whenever $p<q$.

Then

$$
h=\bigvee^{\mathrm{IC}(L)}\{f \in \mathrm{C}(L) \mid f \leq h\} .
$$

Proof. Let

$$
\mathcal{F}=\{f \in \mathrm{C}(L) \mid f \leq h\} .
$$

By $(1), \mathcal{F} \neq \varnothing$. Since $\operatorname{IC}(L)$ is Dedekind complete, the supremum $\bigvee^{\operatorname{IC}(L)} \mathcal{F}$ exists. We shall prove that

$$
\bigvee^{\mathrm{IC}(L)} \mathcal{F}=h .
$$

We only need to show that, for any $h^{\prime} \in \operatorname{IC}(L)$ such that $f \leq h^{\prime}$ for all $f \in \mathcal{F}$, $h \leq h^{\prime}$, i.e.,

(a) $h(p,-) \leq h^{\prime}(p,-)$ for every $p \in \mathbb{Q}$,

(b) $h(-, q) \geq h^{\prime}(-, q)$ for every $q \in \mathbb{Q}$.

(a) We fix $p \in \mathbb{Q}$ and consider $p^{\prime} \in \mathbb{Q}$ such that $p<p^{\prime}$. Since $L$ is completely regular, we obtain $h\left(p^{\prime},-\right)=\bigvee\left\{a \in L \mid a \prec \prec h\left(p^{\prime},-\right)\right\}$. Let $a \in L$ such that $a \prec \prec h\left(p^{\prime},-\right)$. Then there exists a family $\left\{c_{r}: r \in \mathbb{Q} \cap[0,1]\right\} \subseteq L$ such that $a \leq c_{0}, c_{1} \leq h\left(p^{\prime},-\right)$ and $c_{r} \prec c_{s}$ whenever $r<s$. Hence the map $\sigma_{a, p^{\prime}}: \mathbb{Q} \rightarrow L$ given by

$$
\sigma_{a, p^{\prime}}(r)= \begin{cases}0 & \text { if } r>1, \\ c_{1-r} & \text { if } 0 \leq r \leq 1, \\ 1 & \text { if } r<0,\end{cases}
$$

is a scale and generates a $g_{a, p^{\prime}} \in \mathrm{C}(L)$ given by

$$
g_{a, p^{\prime}}(r,-)= \begin{cases}0 & \text { if } r \leq 1, \\ \bigvee_{r^{\prime}>r} c_{1-r^{\prime}} & \text { if } 0 \leq r<1, \\ 1 & \text { if } r<0,\end{cases}
$$

and

$$
g_{a, p^{\prime}}(-, s)= \begin{cases}1 & \text { if } s>1, \\ \bigvee_{s^{\prime}<s} c_{1-s^{\prime}}^{*} & \text { if } 0<s \leq 1, \\ 0 & \text { if } s \leq 0\end{cases}
$$


Evidently $\mathbf{0} \leq g_{a, p^{\prime}} \leq \mathbf{1}$. Let

$$
f_{a, p^{\prime}}=f+\left(\left(\left(\boldsymbol{p}^{\prime}-f\right) \vee \mathbf{0}\right) \cdot g_{a, p^{\prime}}\right) \in \mathrm{C}(L) .
$$

We have $f_{a, p^{\prime}} \leq h$; indeed, for each $r \in \mathbb{Q}$,

$$
\begin{aligned}
f_{a, p^{\prime}}(r,-)= & \bigvee_{r^{\prime} \in \mathbb{Q}} f\left(r-r^{\prime},-\right) \wedge\left(\left(\left(\boldsymbol{p}^{\prime}-f\right) \vee \mathbf{0}\right) \cdot g_{a, p^{\prime}}\right)\left(r^{\prime},-\right) \\
= & \bigvee_{r^{\prime}<0} f\left(r-r^{\prime},-\right) \\
& \vee \bigvee_{r^{\prime} \geq 0} f\left(r-r^{\prime},-\right) \wedge\left(\left(\left(\boldsymbol{p}^{\prime}-f\right) \vee \mathbf{0}\right) \cdot g_{a, p^{\prime}}\right)\left(r^{\prime},-\right) \\
= & f(r,-) \vee \bigvee_{r^{\prime} \geq 0} \bigvee_{r^{\prime \prime}>0} f\left(r-r^{\prime},-\right) \wedge\left(\left(\boldsymbol{p}^{\prime}-f\right) \vee \mathbf{0}\right)\left(r^{\prime \prime},-\right) \\
= & \wedge g_{a, p^{\prime}}\left(\frac{r^{\prime}}{r^{\prime \prime}},-\right) \\
= & f(r,-) \vee \bigvee_{r^{\prime} \geq 0} \bigvee_{r^{\prime \prime}>0} f\left(r-r^{\prime}, p^{\prime}-r^{\prime \prime}\right) \wedge g_{a, p^{\prime}}\left(\frac{r^{\prime}}{r^{\prime \prime}},-\right) \\
& \bigvee_{r^{\prime} \geq 0} f\left(r-r_{r^{\prime}<r^{\prime \prime}<p^{\prime}-r+r^{\prime}} f(r,-) \vee \bigvee_{r^{\prime} \geq 0} \bigvee_{r^{\prime}<r^{\prime \prime}<p^{\prime}-r+r^{\prime}} \bigvee_{r^{\prime \prime \prime}>\frac{r^{\prime}}{r^{\prime \prime}}} f\left(r-r^{\prime \prime}\right) \wedge g_{a, p^{\prime}}\left(\frac{r^{\prime}}{r^{\prime \prime}},-\right)\right.
\end{aligned}
$$

Now, if $r \geq p^{\prime}$, then $p^{\prime}-r+r^{\prime} \leq r^{\prime}$ for each $r^{\prime} \geq 0$ and thus

$$
f_{a, p^{\prime}}(r,-)=f(r,-) \leq h(r,-) .
$$

Otherwise, if $r<p^{\prime}$, then

$$
\begin{aligned}
f_{a, p^{\prime}}(r,-) & \leq f(r,-) \vee \bigvee_{r^{\prime} \geq 0} \bigvee_{r^{\prime}<r^{\prime \prime}<p^{\prime}-r+r^{\prime}} f\left(r-r^{\prime}, p^{\prime}-r^{\prime \prime}\right) \wedge c_{1} \\
& =f(r,-) \vee \bigvee_{r^{\prime} \geq 0} f\left(r-r^{\prime}, p^{\prime}-r^{\prime}\right) \wedge c_{1} \\
& =f(r,-) \vee\left(f\left(-, p^{\prime}\right) \wedge c_{1}\right) \\
& =\left(f(r,-) \vee f\left(-, p^{\prime}\right)\right) \wedge\left(f(r,-) \vee c_{1}\right) \\
& =f(r,-) \vee c_{1} \\
& \leq h(r,-) \vee h\left(p^{\prime},-\right) \\
& =h(r,-) .
\end{aligned}
$$


Hence $f_{a, p^{\prime}}(r,-) \leq h(r,-)$ for every $r \in \mathbb{Q}$ and since $f_{a, p^{\prime}} \in \mathrm{C}(L)$, it follows that $f_{a, p^{\prime}} \leq h$, by Remark 2.6 (2), and we may conclude that $f_{a, p^{\prime}} \in \mathcal{F}$.

Finally, since $p<p^{\prime}$, it follows that

$$
\begin{aligned}
f_{a, p^{\prime}}(p,-) & =f(p,-) \vee \bigvee_{r^{\prime} \geq 0} \bigvee_{r^{\prime}<r^{\prime \prime}<p^{\prime}-p+r^{\prime}} \bigvee_{r^{\prime \prime \prime}>\frac{r^{\prime}}{r^{\prime \prime}}} f\left(p-r^{\prime}, p^{\prime}-r^{\prime \prime}\right) \wedge c_{1-r^{\prime \prime \prime}} \\
& \geq f(p,-) \vee \bigvee_{r^{\prime} \geq 0} \bigvee_{r^{\prime}<r^{\prime \prime}<p^{\prime}-p+r^{\prime}} f\left(p-r^{\prime}, p^{\prime}-r^{\prime \prime}\right) \wedge c_{0} \\
& =f(p,-) \vee \bigvee_{r^{\prime} \geq 0} f\left(p-r^{\prime}, p^{\prime}-r^{\prime}\right) \wedge c_{0} \\
& =f(p,-) \vee\left(f\left(-, p^{\prime}\right) \wedge c_{0}\right) \\
& =\left(f(p,-) \vee f\left(-, p^{\prime}\right)\right) \wedge\left(f(r,-) \vee c_{0}\right) \\
& =f(p,-) \vee c_{0} \\
& \geq c_{0}
\end{aligned}
$$

and thus

$$
a \leq c_{0} \leq f_{a, p^{\prime}}(p,-) \leq h^{\prime}(p,-)
$$

Hence

$$
h(p,-)=\bigvee_{p^{\prime}>p} h\left(p^{\prime},-\right)=\bigvee_{p^{\prime}>p} \bigvee_{a \prec h\left(p^{\prime},-\right)} a \leq h^{\prime}(p,-) .
$$

(b) Using (2) it follows that

$$
\begin{aligned}
h(-, q) & =\bigvee_{s^{\prime}<s} \bigvee_{s<q} h\left(-, s^{\prime}\right) \geq \bigvee_{s<q} h(s,-)^{*} \\
& \geq \bigvee_{s<q} h^{\prime}(s,-)^{*} \geq \bigvee_{s<q} h^{\prime}(-, s)=h^{\prime}(-, q) .
\end{aligned}
$$

Then, it follows from Lemma 3.1 and Remark 2.6 (3) that:

Lemma 3.2. Let $L$ be a completely regular frame and let $h \in \operatorname{IC}(L)$ be such that

(1) $\{g \in \mathrm{C}(L) \mid h \leq g\} \neq \varnothing$,

(2) $h(-, q)^{*} \leq h(p,-)$ whenever $p<q$.

Then

$$
h=\bigwedge^{\mathrm{IC}(L)}\{g \in \mathrm{C}(L) \mid h \leq g\} .
$$


We introduce now the following classes:

$$
\begin{aligned}
& \mathrm{C}(L)^{\vee}=\{h \in \mathrm{IC}(L) \mid \exists f, g \in \mathrm{C}(L): f \leq h \leq g \text { and } \\
& \left.h(p,-)^{*} \leq h(-, q) \text { if } p<q\right\}, \\
& \mathrm{C}(L)^{\wedge}=\{h \in \mathrm{IC}(L) \mid \exists f, g \in \mathrm{C}(L): f \leq h \leq g \text { and } \\
& \left.h(-, q)^{*} \leq h(p,-) \text { if } p<q\right\} \text {, } \\
& \mathrm{C}(L)^{\mathfrak{X}}=\mathrm{C}(L)^{\vee} \cap \mathrm{C}(L)^{\wedge} .
\end{aligned}
$$

The next result is an immediate consequence of Lemmas 3.1 and 3.2.

Proposition 3.3. Let $L$ be a completely regular frame and let $h \in \mathrm{C}(L)^{\mathrm{W}}$. Then

$$
h=\bigvee^{\mathrm{IC}(L)}\{f \in \mathrm{C}(L) \mid f \leq h\}=\bigwedge^{\mathrm{IC}(L)}\{g \in \mathrm{C}(L) \mid h \leq g\} .
$$

The following diagram depicts the inclusions between those classes (each arrow represents a strict inclusion):

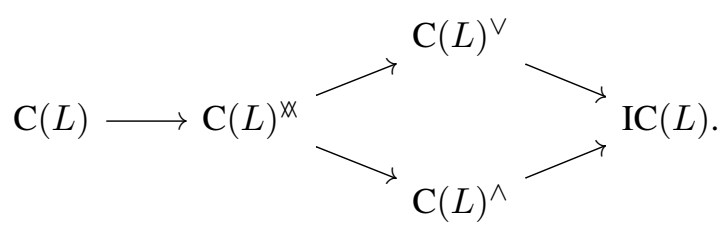

The only non trivial inclusion, that is, $\mathrm{C}(L) \subseteq \mathrm{C}(L)^{\mathrm{x}}$, follows from the fact that $h(p,-) \vee h(-, q)=1$ implies $h(p,-)^{*} \leq h(-, q)$ and $h(-, q)^{*} \leq h(p,-)$. Further, the inclusions are strict. Indeed, for each $a, b \in L$ such that $a \wedge b=0$ recall the bounded $\chi_{a, b}$ from Example 2.7. Then:

(1) $\chi_{a, b} \in \mathrm{C}(L)^{\vee}$ if and only if $a^{*}=b$,

(2) $\chi_{a, b} \in \mathrm{C}(L)^{\wedge}$ if and only if $b^{*}=a$,

(3) $\chi_{a, b} \in \mathrm{C}(L)^{\mathcal{X}}$ if and only if $a^{*}=b$ and $b^{*}=a$, i.e., if and only if $a$ is regular and $b=a^{*}$.

Consequently,

- if $a$ is regular but not complemented, then $\chi_{a, a^{*}} \in \mathrm{C}(L)^{\not X} \backslash \mathrm{C}(L)$,

- if $a^{*}=b$ but $b^{*} \neq a$, then $\chi_{a, a^{*}} \in \mathrm{C}(L)^{\vee} \backslash \mathrm{C}(L)^{\wedge}$ (for instance, take $L=\mathcal{O} \mathbb{R}$, $a=\mathbb{R} \backslash\{0\}$ and $b=\varnothing)$,

- if $b^{*}=a$ but $a^{*} \neq b$, then $\chi_{b^{*}, b} \in \mathrm{C}(L)^{\wedge} \backslash \mathrm{C}(L)^{\vee}$,

- if $a^{*} \neq b$ and $b^{*} \neq a$, then $\chi_{a, b} \in \mathrm{IC}(L) \backslash\left(\mathrm{C}(L)^{\vee} \cup \mathrm{C}(L)^{\wedge}\right)$ (for instance, take $a=b=0)$. 
Remark 3.4. The order reversing isomorphism $-(\cdot): \operatorname{IC}(L) \rightarrow \operatorname{IC}(L)$ introduced in Remarks 2.6 induces an isomorphism from $\mathrm{C}(L)^{\vee}$ onto $\mathrm{C}(L)^{\wedge}$ (and hence an isomorphism from $\mathrm{C}(L)^{\text {WX }}$ onto $\left.\mathrm{C}(L)^{\text {XX }}\right)$.

Proposition 3.5. The class $\mathrm{C}(L)^{\vee}$ is closed under non-void bounded suprema and $\mathrm{C}(L)^{\wedge}$ is closed under non-void bounded infima.

Proof. Let $\left\{h_{i}\right\}_{i \in I} \subseteq \mathrm{C}(L)^{\vee}$ and $h \in \mathrm{C}(L)^{\vee}$ such that

$$
h_{i} \leq h \quad \text { for all } i \in I .
$$

On one hand, since $\operatorname{IC}(L)$ is Dedekind complete, the supremum $\bigvee_{i \in I}^{\operatorname{IC}(L)} h_{i}$ exists and it is given by

$$
\left(\bigvee_{i \in I}^{\operatorname{IC}(L)} h_{i}\right)(p,-)=\bigvee_{i \in I} h_{i}(p,-) \text { and }\left(\bigvee_{i \in I}^{\operatorname{IC}(L)} h_{i}\right)(-, q)=\bigvee_{s<q} \bigwedge_{i \in I} h_{i}(-, s)
$$

for every $p, q \in \mathbb{Q}$. On the other hand, for each $i \in I$, since $h_{i} \in \mathrm{C}(L)^{\vee}$, there exists an $f_{i} \in \mathrm{C}(L)$ such that $f_{i} \leq h_{i}$, and since $h \in \mathrm{C}(L)^{\vee}$, there exists $g \in \mathrm{C}(L)$ such that $h \leq g$. Consequently,

$$
f_{i} \leq h_{i} \leq \bigvee_{i \in I}^{\mathrm{IC}(L)} h_{i} \leq g
$$

Further, let $p<q$ in $\mathbb{Q}$ and $p<r<q$. Then

$$
\left(\left(\bigvee_{i \in I}^{\operatorname{IC}(L)} h_{i}\right)(p,-)\right)^{*}=\bigwedge_{i \in I} h_{i}(p,-)^{*} \leq \bigwedge_{i \in I} h_{i}(-, r) \leq\left(\bigvee_{i \in I}^{\operatorname{IC}(L)} h_{i}\right)(-, q),
$$

which shows that

$$
\bigvee_{i \in I}^{\mathrm{IC}(L)} h_{i} \in \mathrm{C}(L)^{\vee} .
$$

The second assertion follows immediately by Remark 3.4.

Finally, we establish the main result of the paper.

Theorem 3.6. The class $\mathrm{C}(L)^{\mathrm{W}}$ is Dedekind complete.

Proof. (a) Let $\left\{h_{i}\right\}_{i \in I} \subseteq \mathrm{C}(L)^{\mathbb{W}}$ and $h \in \mathrm{C}(L)^{\mathrm{WX}}$ such that

$$
h_{i} \leq h \quad \text { for all } i \in I .
$$


For each $r, s \in \mathbb{Q}$ we define $h_{\vee}: \mathfrak{L}(\mathbb{I} \mathbb{R}) \rightarrow L$ on generators by

$$
h_{\vee}(r,-)=\bigvee_{p>r}\left(\bigwedge_{i \in I} h_{i}(p,-)^{*}\right)^{*} \text { and } h_{\vee}(-, s)=\bigvee_{q<s} \bigwedge_{i \in I} h_{i}(q,-)^{*}
$$

This is a frame homomorphism since it turns the defining relations (r1) and (r3)(r6) of $\mathfrak{L}(\mathbb{I} \mathbb{R})$ into identities in $L$ :

(r1) whenever $r \geq s$,

$$
h_{\vee}(r,-) \wedge h_{\vee}(-, s) \leq\left(\bigwedge_{i \in I} h_{i}(r,-)^{*}\right)^{*} \wedge \bigwedge_{i \in I} h_{i}(r,-)^{*}=0 .
$$

(r3) for each $r \in \mathbb{Q}$,

$$
\bigvee_{s>r} h_{\vee}(s,-)=\bigvee_{s>r} \bigvee_{p>s}\left(\bigwedge_{i \in I} h_{i}(p,-)^{*}\right)^{*}=h_{\vee}(r,-) .
$$

(r4) for each $r \in \mathbb{Q}$,

$$
\bigvee_{s<r} h_{\vee}(-, s)=\bigvee_{s<r} \bigvee_{q<s} \bigwedge_{i \in I} h_{i}(q,-)^{*}=\bigvee_{q<r} \bigwedge_{i \in I} h_{i}(q,-)^{*}=h_{\vee}(-, r) .
$$

(r5) we have

$$
\bigvee_{r \in \mathbb{Q}} h_{\vee}(r,-)=\bigvee_{r \in \mathbb{Q}} \bigvee_{p>r}\left(\bigwedge_{i \in I} h_{i}(p,-)^{*}\right)^{*} \geq \bigvee_{p \in \mathbb{Q}} \bigvee_{i \in I} h_{i}(p,-)=1
$$

(r6) we have

$$
\bigvee_{s \in \mathbb{Q}} h_{\vee}(-, s)=\bigvee_{s \in \mathbb{Q}} \bigvee_{q<s} \bigwedge_{i \in I} h_{i}(q,-)^{*}=\bigvee_{q \in \mathbb{Q}} h(q,-)^{*} \geq \bigvee_{q \in \mathbb{Q}} h(-, q)=1 .
$$

Moreover, for each $r<s$ in $\mathbb{Q}$ and $r<t<s$,

$$
\begin{aligned}
h_{\vee}(r,-)^{*} & =\bigwedge_{p>r}\left(\bigwedge_{i \in I} h_{i}(p,-)^{*}\right)^{* *}=\bigwedge_{p>r}\left(\bigvee_{i \in I} h_{i}(p,-)\right)^{* * *} \\
& \leq\left(\bigvee_{i \in I} h_{i}(t,-)\right)^{*} \leq h_{\vee}(-, s), \\
h_{\vee}(-, s)^{*} & =\bigwedge_{q<s}\left(\bigwedge_{i \in I} h_{i}(q,-)^{*}\right)^{*} \\
& \leq\left(\bigwedge_{i \in I} h_{i}(t,-)^{*}\right)^{*} \leq h_{\vee}(r,-) .
\end{aligned}
$$


Further, for each $r, s \in \mathbb{Q}$ and $i \in I$, we have

$$
\begin{aligned}
h_{i}(r,-) & =\bigvee_{p>r} h_{i}(p,-) \leq \bigvee_{p>r} h_{i}(p,-)^{* *} \\
& \leq h_{\vee}(r,-) \leq \bigvee_{p>r} h(p,-)^{* *} \leq h(r,-), \\
h(-, s) & =\bigvee_{q<s} h(-, q) \leq \bigvee_{q<s} h(q,-)^{*} \\
& \leq h_{\vee}(-, s) \leq \bigvee_{q<s} h_{i}(-, q)=h_{i}(-, s)
\end{aligned}
$$

and thus $h_{i} \leq h_{\vee} \leq h$ for all $i \in I$. Since $h_{i} \in \mathrm{C}(L)^{\vee}$, there exists an $f_{i} \in \mathrm{C}(L)$ such that $f_{i} \leq h_{i}$, and since $h \in \mathrm{C}(L)^{\wedge}$, there exists a $g \in \mathrm{C}(L)$ such that $h \leq g$. Consequently $h_{\vee} \in \mathrm{C}(L)^{\mathfrak{X}}$. Finally, if $g \in \mathrm{C}(L)^{\mathfrak{X}}$ is such that $h_{i} \leq g$ for every $i \in I$, then

$$
\begin{aligned}
& g(r,-) \geq \bigvee_{p>r} g(p,-)^{* *} \geq \bigvee_{p>r}\left(\bigvee_{i \in I} h_{i}(p,-)\right)^{* *}=h_{\vee}(r,-), \\
& g(-, s)=\bigvee_{q<s} g(-, q) \leq \bigvee_{q<s} \bigwedge_{i \in I} h_{i}(-, q) \leq \bigvee_{q<s} \bigwedge_{i \in I} h_{i}(q,-)^{*}=h_{\vee}(-, s)
\end{aligned}
$$

for every $r, s \in \mathbb{Q}$ and therefore $h_{\vee} \leq g$. Hence $h_{\vee}$ is the supremum of $\left\{h_{i}\right\}_{i \in I}$ in $\mathrm{C}(L)^{\text {XX }}$.

(b) If $\left\{h_{i}\right\}_{i \in I} \subseteq \mathrm{C}(L)^{\mathscr{W}}$ and $h \in \mathrm{C}(L)^{\mathscr{W}}$ is such that $h \leq h_{i}$ for all $i \in I$, then

$$
\left\{-h_{i}\right\}_{i \in I} \subseteq \mathrm{C}(L)^{X \mathbb{X}}
$$

and $-h \in \mathrm{C}(L)^{\mathrm{x}}$ is such that $-h_{i} \leq-h$. By (a), the supremum $\bigvee_{i \in I}^{\mathrm{C}(L)^{\mathrm{W}}}\left(-h_{i}\right)$ exists. It is easy to check that

$$
\bigwedge_{i \in I}^{\mathrm{C}(L)^{\mathrm{N}}} h_{i}=-\bigvee_{i \in I}^{\mathrm{C}(L)^{\mathrm{N}}}\left(-h_{i}\right) .
$$

As an immediate consequence of Proposition 3.3 and Theorem 3.6 we have:

Corollary 3.7. Let $L$ be a frame. Then the Dedekind completion $D(\mathrm{C}(L))$ of $\mathrm{C}(L)$ coincides with $\mathrm{C}(L)^{\mathrm{x}}$, i.e.,

$$
\begin{gathered}
D(\mathrm{C}(L))=\{h \in \mathrm{IC}(L) \mid \text { (a) there exist } f, g \in \mathrm{C}(L) \text { such that } f \leq h \leq g, \\
\text { (b) } h(p,-)^{*} \leq h(-, q) \text { and } h(-, q)^{*} \leq h(p,-) \\
\text { for any } p<q\} .
\end{gathered}
$$


We conclude this section with the result that the elements of the completion $D(\mathrm{C}(L))$ can be alternatively described as some maximal elements of $\operatorname{IC}(L)$ with respect to the following partial order on $\operatorname{IC}(L)$ :

$$
f \sqsubseteq g \quad \text { iff } \quad f(r,-) \leq g(r,-) \text { and } f(-, r) \leq g(-, r) \text { for all } r \in \mathbb{Q} .
$$

We shall also describe the classes $\mathrm{C}(L)^{\vee}, \mathrm{C}(L)^{\wedge}$ and $\mathrm{C}(L)^{\text {xx }}$ in terms of the partial order $\sqsubseteq$.

Proposition 3.8. The following are equivalent for any $h \in \operatorname{IC}(L)$.

(i) $h(p,-)^{*} \leq h(-, q)$ whenever $p<q$ in $\mathbb{Q}$.

(ii) $g(-, r)=h(-, r)$ for all $r \in \mathbb{Q}$ and all $g \in \operatorname{IC}(L)$ such that $h \sqsubseteq g$.

Proof. In order to check that (i) $\Rightarrow$ (ii), let $g \in \operatorname{IC}(L)$ such that $h \sqsubseteq g$. By (i), $g(p,-)^{*} \leq h(p,-)^{*} \leq h(-, q) \leq g(-, q)$ for all $p<q$ in $\mathbb{Q}$. Consequently,

$$
g(-, q)=\bigvee_{p<q} g(p,-)^{*} \quad \text { for all } q \in \mathbb{Q} .
$$

Thus we get

$$
g(-, q)=\bigvee_{p<q} g(p,-)^{*} \leq h(-, q) \leq g(-, q)
$$

and so $g(-, q)=h(-, q)$.

For the reverse implication let $g \in \operatorname{IC}(L)$ be defined as follows:

$$
g(r,-)=\bigvee_{s>r} h(s,-)^{* *} \text { and } g(-, r)=\bigvee_{s<r} h(s,-)^{*}
$$

It is straightforward to check that $g$ is indeed a partial continuous functions and that $h \sqsubseteq g$. Therefore, by hypothesis, $h(-, r)=g(-, r)$ for all $r \in \mathbb{Q}$. Consequently,

$$
\bigvee_{s<r} h(s,-)^{*}=h(-, r),
$$

which implies $h(s,-)^{*} \leq h(-, r)$ for all $s<r$ in $\mathbb{Q}$.

Proposition 3.9. The following are equivalent for any $h \in \operatorname{IC}(L)$.

(i) $h(-, q)^{*} \leq h(p,-)$ whenever $p<q$ in $\mathbb{Q}$.

(ii) $g(r,-)=h(r,-)$ for all $r \in \mathbb{Q}$ and all $g \in \operatorname{IC}(L)$ such that $h \sqsubseteq g$. 
Proof. Clearly,

$$
h(-, q)^{*} \leq h(p,-) \quad \text { for all } p<q
$$

if and only if

$$
(-h(-q,-))^{*} \leq-h(-,-p) \text { for all }-q<-p,
$$

which is equivalent to $-g(-, r)=-h(-, r)$ for all $r \in \mathbb{Q}$ and all $g \in \operatorname{IC}(L)$ such that $-h \sqsubseteq-g$ (by Proposition 3.8).

Then we may conclude that the elements $h$ of $\mathrm{C}(L)^{\mathfrak{X}}$ are precisely the maximal elements of $(\operatorname{IC}(L), \sqsubseteq)$ for which there exist $f, g \in \mathrm{C}(L)$ satisfying $f \leq h \leq g$ :

Corollary 3.10. Let $L$ be a frame. Then

$$
\begin{gathered}
\mathrm{C}(L)^{\mathfrak{W}}=\{h \in \mathrm{IC}(L) \mid \\
\mid \text { (a) there exist } f, g \in \mathrm{C}(L) \text { such that } f \leq h \leq g, \\
\text { (b) } \left.h \sqsubseteq h^{\prime} \in \mathrm{IC}(L) \Longrightarrow h=h^{\prime}\right\} .
\end{gathered}
$$

Remark 3.11. One might wonder whether the operations of the algebra $\mathrm{C}(L)$ (described in Subsection 1.3) can be extended to $C^{\not X}(L)$ in such a way that $C^{\text {X }}(L)$ becomes a lattice-ordered ring. This is indeed true: using the techniques introduced in [13], the operations on $\mathrm{C}(L)$ can be easily extended to $\operatorname{IC}(L)$ (since none of $\mathrm{C}(L)^{\vee}, \mathrm{C}(L)^{\wedge}$, or $\operatorname{IC}(L)$ is even a group, one may view this fact as a happy accident). The lengthy details of the proof, however, go beyond the scope of this paper and will be treated elsewhere.

\section{The bounded case}

In this section we show that if we restrict the preceding statements to bounded functions, most results remain essentially the same.

Proposition 4.1. The class $\mathrm{IC}^{*}(L)$ is Dedekind complete.

Proof. Let $\left\{h_{i}\right\}_{i \in I} \subseteq \mathrm{IC}^{*}(L)$ and $h \in \mathrm{IC}^{*}(L)$ such that

$$
h_{i} \leq h \quad \text { for all } i \in I .
$$

Since $\operatorname{IC}(L)$ is Dedekind complete, there exists $\bigvee_{i \in I}^{\mathrm{IC}(L)} h_{i}$. Let $j \in I$. Then both $h_{j}$ and $h$ are bounded and so there are $p, q \in \mathbb{Q}$ such that

$$
\boldsymbol{p} \leq h_{j} \leq \bigvee_{i \in I}^{\mathrm{IC}(L)} h_{i} \leq h \leq \boldsymbol{q} .
$$


Consequently,

$$
\bigvee_{i \in I}^{\mathrm{IC}^{*}(L)} h_{i}=\bigvee_{i \in I}^{\mathrm{IC}(L)} h_{i} .
$$

Dually, if $h \leq h_{i}$ for all $i \in I$ and some $h \in \mathrm{IC}^{*}(L)$, one has

$$
\bigwedge_{i \in I}^{\mathrm{IC}^{*}(L)} h_{i}=\bigwedge_{i \in I}^{\mathrm{IC}(L)} h_{i} .
$$

Let

$$
\begin{aligned}
& \mathrm{C}^{*}(L)^{\vee}=\mathrm{C}(L)^{\vee} \cap \mathrm{IC}^{*}(L), \\
& \mathrm{C}^{*}(L)^{\wedge}=\mathrm{C}(L)^{\wedge} \cap \mathrm{IC}^{*}(L), \\
& \mathrm{C}^{*}(L)^{X}=\mathrm{C}(L)^{x} \cap \mathrm{IC}^{*}(L) .
\end{aligned}
$$

Proposition 4.2. For any completely regular frame $L$ and $h \in \mathrm{C}^{*}(L)^{\vee}$,

$$
h=\bigvee^{\mathrm{IC}(L)}\left\{f \in \mathrm{C}^{*}(L) \mid f \leq h\right\} .
$$

Proof. Since $h$ is bounded, there exist $p, q \in \mathbb{Q}$ such that $\boldsymbol{p} \leq h \leq \boldsymbol{q}$. Note that $f \vee \boldsymbol{p} \in \mathrm{C}^{*}(L)$ for any $f \in \mathrm{C}(L)$ such that $f \leq h$, since $\boldsymbol{p} \leq f \vee \boldsymbol{p} \leq \boldsymbol{q}$. Then, by Lemma 3.1, one has

$$
\begin{aligned}
h & =\bigvee\{f \in \mathrm{C}(L) \mid f \leq h\} \\
& \leq \bigvee\{f \vee \boldsymbol{I C} \mid f \in \mathrm{C}(L), f \leq h\} \\
& \leq \bigvee\left\{f \in \mathrm{C}^{*}(L) \mid f \leq h\right\} \leq h,
\end{aligned}
$$

and, consequently,

$$
h=\bigvee^{\operatorname{IC}(L)}\left\{f \in \mathrm{C}^{*}(L) \mid f \leq h\right\} .
$$

Proposition 4.3. Let $L$ be a completely regular frame and $h \in \mathrm{C}^{*}(L)^{\wedge}$. Then

$$
h=\bigwedge^{\mathrm{IC}(L)}\left\{g \in \mathrm{C}^{*}(L) \mid h \leq g\right\} .
$$

Proof. It follows from Lemma 3.2, in a similar way as the preceding proposition follows from Lemma 3.1 . 
Corollary 4.4. Let $L$ be a completely regular frame and $h \in \mathrm{C}^{*}(L)^{\mathrm{Xx}}$. Then

$$
h=\bigvee^{\mathrm{IC}^{*}(L)}\left\{f \in \mathrm{C}^{*}(L) \mid f \leq h\right\}=\bigwedge^{\mathrm{IC}^{*}(L)}\left\{g \in \mathrm{C}^{*}(L) \mid h \leq g\right\} .
$$

Proposition 4.5. The class $\mathrm{C}^{*}(L)^{\vee}$ is closed under non-void bounded suprema and $\mathrm{C}^{*}(L)^{\wedge}$ is closed under non-void bounded infima.

Proof. Let $\left\{h_{i}\right\}_{i \in I} \subseteq \mathrm{C}^{*}(L)^{\vee}$ and $h \in \mathrm{C}^{*}(L)^{\vee}$ such that

$$
h_{i} \leq h \quad \text { for all } i \in I .
$$

Since $\mathrm{C}(L)^{\vee}$ is closed under non-void bounded suprema, there exists $\bigvee_{i \in I}^{\mathrm{C}(L)^{\vee}} h_{i}$. As $h$ is bounded from above and each $h_{i}$ is bounded from below, we get

$$
\bigvee_{i \in I}^{\mathrm{C}(L)^{\vee}} h_{i} \in \mathrm{C}^{*}(L)^{\vee}
$$

and thus $\mathrm{C}^{*}(L)^{\vee}$ is closed under non-void bounded suprema.

Proposition 4.6. For any completely regular frame $L, \mathrm{C}^{*}(L)^{\mathrm{W}}$ is Dedekind complete.

Proof. Let $\left\{h_{i}\right\}_{i \in I} \subseteq \mathrm{C}^{*}(L)^{\text {WX }}$ and $h \in \mathrm{C}^{*}(L)^{\mathscr{W}}$ such that

$$
h_{i} \leq h \quad \text { for all } i \in I .
$$

Then, since $\mathrm{C}(L)^{\mathrm{Wx}}$ is Dedekind complete, $\bigvee_{i \in I}^{\mathrm{C}(L)^{\mathrm{Wx}}} h_{i}$ exists, and as each $h_{i}$ is bounded from below and $h$ is bounded from above, it is bounded. Consequently,

$$
\bigvee_{i \in I}^{\mathrm{C}^{*}(L)^{\mathrm{N}}} h_{i}=\bigvee_{i \in I}^{\mathrm{C}(L)^{\mathrm{N}}} h_{i} .
$$

The second assertion follows in a similar way.

Corollary 4.7. For any completely regular frame $L, \mathrm{C}^{*}(L)^{\mathrm{Wx}}$ is the Dedekind completion of $\mathrm{C}^{*}(L)$.

We close this section with a corollary that augments a characterization of Banaschewski-Hong [7, Proposition 1]. 
Corollary 4.8. For any completely regular frame $L$, the following are equivalent.

(1) L is extremally disconnected.

(2) $\mathrm{C}(L)=\mathrm{C}(L)^{\mathrm{XX}}$.

(3) $\mathrm{C}(L)$ is Dedekind complete.

(4) $\mathrm{C}(L)$ is closed under non-void bounded suprema.

(5) $\mathrm{C}^{*}(L)=\mathrm{C}^{*}(L)^{\text {Xx}}$.

(6) $\mathrm{C}^{*}(L)$ is Dedekind complete.

(7) $\mathrm{C}^{*}(L)$ is closed under non-void bounded suprema.

Proof. (1) $\Rightarrow(2)$ Let $L$ be extremally disconnected, $h \in \mathrm{C}(L)^{\mathrm{XX}}$ and $p<r<q$. Then $h(r,-)^{*} \leq h(-, q)$ and $h(r,-)^{* *} \leq h(-, r)^{*} \leq h(p,-)$. Hence

$$
h(p,-) \vee h(-, q) \geq h(r,-)^{* *} \vee h(r,-)^{*}=1
$$

Consequently, $\mathrm{C}(L)=\mathrm{C}(L)^{\text {xx }}$.

(3) $\Rightarrow$ (1) For each element $a \in L$, let

$$
\mathcal{F}_{a}=\left\{f \in \mathrm{C}(L) \mid f \leq \chi_{a^{*}, a^{* *}}\right\} \quad \text { and } \quad \mathcal{G}_{a}=\left\{g \in \mathrm{C}(L) \mid \chi_{a^{*}, a^{* *}} \leq g\right\}
$$

By Lemmas 3.1 and 3.2,

$$
\chi_{a^{*}, a^{* *}}=\bigvee^{\mathrm{IC}(L)} \mathcal{F}_{a}=\bigwedge^{\mathrm{IC}(L)} \mathcal{G}_{a}
$$

On the other hand, since $\mathbf{0} \in \mathcal{F}_{a}, \mathbf{1} \in \mathcal{G}_{a}, f \leq \mathbf{1}$ for all $f \in \mathcal{F}_{a}$ and $\mathbf{0} \leq g$ for all $g \in \mathcal{G}_{a}$,

$$
\bigvee^{\mathrm{C}(L)} \mathcal{F}_{a} \text { and } \bigwedge^{\mathrm{C}(L)} \mathcal{G}_{a}
$$

do exist. Therefore

$$
\chi_{a^{*}, a^{* *}}=\bigvee^{\mathrm{IC}(L)} \mathcal{F}_{a} \leq \bigvee^{\mathrm{C}(L)} \mathcal{F}_{a} \leq \bigwedge^{\mathrm{C}(L)} \mathcal{G}_{a} \leq \bigwedge^{\mathrm{IC}(L)} \mathcal{G}_{a}=\chi_{a^{*}, a^{* *}}
$$

and we may conclude that $\chi_{a^{*}, a^{* *}} \in \mathrm{C}(L)$, that is, $a^{*} \vee a^{* *}=1$.

Finally, the implication (2) $\Rightarrow(3)$ follows from Theorem 3.6, the equivalence $(3) \Leftrightarrow(4)$ is obvious and the equivalences $(1) \Leftrightarrow(5) \Leftrightarrow(6) \Leftrightarrow(7)$ can be proved in a similar way. 


\section{The integer-valued case}

Recall from [4] and [7] that the ring $\mathfrak{Z} L$ of integer-valued continuous functions on a frame $L$ has as its elements the maps $\alpha, \beta, \gamma, \ldots: \mathbb{Z} \rightarrow L$ such that

$$
\alpha(n) \wedge \alpha(m)=0 \quad \text { for } n \neq m \quad \text { and } \quad \bigvee\{\alpha(n) \mid n \in \mathbb{Z}\}=1 .
$$

The elements of $\mathfrak{Z} L$ can be easily identified with those elements of $f \in \mathrm{C}(L)$ such that

$$
f(p,-)=f(\lfloor p\rfloor,-) \text { and } f(-, q)=f(-,\lceil q\rceil) \quad \text { for all } p, q \in \mathbb{Q} \quad(\mathbb{Z} \text {-valued })
$$

(where $\lfloor p\rfloor$ denotes the biggest integer $\leq p$ and $\lceil q\rceil$ the smallest integer $\geq q$ ). Denoting the subclass of $\mathrm{C}(L)$ of all $\mathbb{Z}$-valued functions by $\mathrm{C}(L, \mathbb{Z})$, the correspondence $\mathfrak{Z} L \simeq \mathrm{C}(L, Z)$ is given by

$$
\begin{aligned}
\alpha \in \mathfrak{Z} L & \mapsto f_{\alpha}(p,-)=\bigvee\{\alpha(n) \mid p<n\}, f_{\alpha}(-, q)=\bigvee\{\alpha(n) \mid n<q\}, \\
f \in \mathrm{C}(L, Z) & \mapsto \alpha_{f}(n)=f(n-1,-) \wedge f(-, n+1) .
\end{aligned}
$$

From this it follows that the Dedekind completion of $\mathfrak{Z} L$ is isomorphic to the Dedekind completion of $\mathrm{C}(L, \mathbb{Z})$, which is included in $\mathrm{C}(L)^{\mathrm{X}}$.

In the same vein, we shall also denote by $\operatorname{IC}(L, \mathbb{Z}), \mathrm{C}(L, \mathbb{Z})^{\vee}, \mathrm{C}(L, \mathbb{Z})^{\wedge}$ and $\mathrm{C}(L, \mathbb{Z})^{\mathcal{X}}$ the $\mathbb{Z}$-valued subsets of $\operatorname{IC}(L), \mathrm{C}(L)^{\vee}, \mathrm{C}(L)^{\wedge}$ and $\mathrm{C}(L)^{\mathfrak{X}}$, respectively.

Example 5.1. The bounded continuous partial real function $\chi_{a, b}$ (where $a, b \in L$, $a \wedge b=0$ ) from Example 2.7 is clearly $\mathbb{Z}$-valued. Moreover:

(1) $\chi_{a, b} \in \operatorname{IC}(L, \mathbb{Z})$.

(2) $\chi_{a, b} \in \mathrm{C}(L, \mathbb{Z})^{\vee}$ if and only if $a^{*}=b$.

(3) $\chi_{a, b} \in \mathrm{C}(L, \mathbb{Z})^{\wedge}$ if and only if $b^{*}=a$.

(4) $\chi_{a, b} \in \mathrm{C}(L, \mathbb{Z})^{\mathbb{N}}$ if and only if $a^{*}=b$ and $b^{*}=a$, i.e., if and only if $a$ is regular and $b=a^{*}$.

(5) $\chi_{a, b} \in \mathbf{C}(L, \mathbb{Z})$ if and only if $a$ is complemented with complement $b$.

Proposition 5.2. The class $\mathrm{IC}(L, \mathbb{Z})$ is Dedekind complete.

Proof. Let $\left\{h_{i}\right\}_{i \in I} \subseteq \operatorname{IC}(L, \mathbb{Z}), h \in \operatorname{IC}(L, \mathbb{Z})$, such that

$$
h_{i} \leq h \quad \text { for all } i \in I .
$$


Since $\operatorname{IC}(L)$ is Dedekind complete, there exists $\bigvee_{i \in I}^{\operatorname{IC}(L)} h_{i}$ in $\operatorname{IC}(L)$. In addition, for each $r, s \in \mathbb{Q}$,

$$
\begin{aligned}
\bigvee_{i \in I}^{\mathrm{IC}(L)} h_{i}(r,-) & =\bigvee_{i \in I} h_{i}(r,-)=\bigvee_{i \in I} h_{i}(\lfloor r\rfloor,-)=h_{\vee}(\lfloor r\rfloor,-), \\
\bigvee_{i \in I}^{\mathrm{IC}(L)} h_{i}(-, s) & =\bigvee_{q<s} \bigwedge_{i \in I} h_{i}(-, q)=\bigvee_{q<s} \bigwedge_{i \in I} h_{i}(-,\lceil q\rceil) \\
& =\bigvee_{q<\lceil s\rceil} \bigwedge_{i \in I} h_{i}(-,\lceil q\rceil)=h_{\vee}(-,\lceil s\rceil)
\end{aligned}
$$

which ensures that $\bigvee_{i \in I} h_{i}$ is $\mathbb{Z}$-valued. $\quad \operatorname{IC}(L)$

Dually, if $h \leq h_{i}$ for all $i \in I$, one gets that $\bigwedge_{i \in I} h_{i}$ is $\mathbb{Z}$-valued.

Proposition 5.3. Let $L$ be a zero-dimensional frame and let $h \in \mathrm{C}(L, \mathbb{Z})^{\vee}$. Then

$$
h=\bigvee^{\mathrm{IC}(L, \mathbb{Z})}\{f \in \mathrm{C}(L, \mathbb{Z}) \mid f \leq h\} .
$$

Proof. Let

$$
\mathcal{F}=\{f \in \mathbf{C}(L, \mathbb{Z}) \mid f \leq h\}
$$

Since $\operatorname{IC}(L, \mathbb{Z})$ is Dedekind complete, $\bigvee^{\mathrm{IC}(L, \mathbb{Z})} \mathcal{F}$ exists. We shall prove that

$$
\bigvee^{\mathrm{IC}(L, \mathbb{Z})} \mathcal{F}=h .
$$

For that we only need to check that $h \leq h^{\prime}$ for any $h^{\prime} \in \operatorname{IC}(L, \mathbb{Z})$ such that $f \leq h^{\prime}$ for all $f \in \mathcal{F}$, i.e.,

(a) $h(p,-) \leq h^{\prime}(p,-)$ for every $p \in \mathbb{Q}$,

(b) $h(-, q) \geq h^{\prime}(-, q)$ for every $q \in \mathbb{Q}$.

(a) Fix $p \in \mathbb{Q}$, let $n=\lfloor p\rfloor$ and $f \in \mathbf{C}(L, \mathbb{Z})$ such that $f \leq h$. Since $L$ is zerodimensional, we get

$$
h(p,-)=h(n,-)=\bigvee\{a \in L \mid a \text { is complemented and } a \leq h(n,-)\} .
$$

For each such complemented $a$, define $\sigma_{a, n}: \mathbb{Q} \rightarrow L$ by

$$
\sigma_{a, n}(r)= \begin{cases}f(r,-) & \text { if } r \geq n+1 \\ f(r,-) \vee a & \text { if } r<n+1\end{cases}
$$


This is a scale in $L$. Indeed,

$$
\begin{aligned}
& \bigvee_{r \in \mathbb{Q}} \sigma_{a, n}(r) \geq \bigvee_{r \in \mathbb{Q}} f(r,-)=1, \\
& \bigvee_{r \in \mathbb{Q}} \sigma_{a, n}(r)^{*} \geq \bigvee_{r \geq n+1} f(r,-)^{*}=1
\end{aligned}
$$

and if $r, s \in \mathbb{Q}$ are such that $r<s$, then

$$
\sigma_{a, n}(r) \vee \sigma_{a, n}(s)^{*}= \begin{cases}f(r,-) \vee f(s,-)^{*}=1 & \text { if } r, s \geq n+1, \\ f(r,-) \vee a \vee f(s,-)^{*}=1 & \text { if } s \geq n+1>r, \\ f(r,-) \vee a \vee\left(f(s,-)^{*} \wedge a^{*}\right) & \\ \geq\left(f(r,-) \vee f(s,-)^{*}\right) \wedge\left(a \vee a^{*}\right)=1 & \text { if } r, s<n+1 .\end{cases}
$$

Consequently, it defines an $f_{a, n} \in \mathrm{C}(L)$ by

$$
f_{a, n}(r,-)= \begin{cases}f(r,-) & \text { if } r \geq n+1, \\ f(r,-) \vee a & \text { if } r<n+1\end{cases}
$$

and

$$
f_{a, n}(-, s)= \begin{cases}f(-, s) & \text { if } s>n+1, \\ f(-, s) \wedge a^{*} & \text { if } s \leq n+1 .\end{cases}
$$

It is easy to check that $f_{a, n}$ is $\mathbb{Z}$-valued. Moreover, $f_{a, n} \leq h$ :

- If $r \geq n+1$, then $f_{a, n}(r,-)=f(r,-) \leq h(r,-)$.

- If $r<n+1$, then $\lfloor r\rfloor \leq n$ and so

$$
\begin{aligned}
f_{a, n}(r,-) & =f(r,-) \vee a \leq h(r,-) \vee h(n,-)=h(\lfloor r\rfloor,-) \vee h(n,-) \\
& =h(\lfloor r\rfloor,-)=h(r,-) .
\end{aligned}
$$

Hence $f_{a, n}(r,-) \leq h(r,-)$ for each $r \in \mathbb{Q}$ and since $f_{a, n} \in \mathrm{C}(L)$, it follows that $f_{a, n} \leq h$. We conclude that $f_{a, n} \in \mathcal{F}$.

Finally, we have also that

$$
a \leq f(n,-) \vee a=f_{a, n}(n,-) \leq h^{\prime}(n,-)=h^{\prime}(p,-) .
$$

Hence

$$
\begin{aligned}
h(p,-) & =h(n,-) \\
& =\bigvee\{a \in L \mid a \text { is complemented and } a \leq h(n,-)\} \\
& \leq h^{\prime}(p,-) .
\end{aligned}
$$


(b) Since $h \in \mathrm{C}(L, \mathbb{Z})^{\vee}$, we have

$$
\begin{aligned}
h(-, q) & =\bigvee_{s^{\prime}<s} \bigvee_{s<q} h\left(-, s^{\prime}\right) \geq \bigvee_{s<q} h(s,-)^{*} \geq \bigvee_{s<q} h^{\prime}(s,-)^{*} \\
& \geq \bigvee_{s<q} h^{\prime}(-, s)=h^{\prime}(-, q) .
\end{aligned}
$$

Then

$$
\bigvee^{\mathrm{IC}(L, \mathbb{Z})} \mathcal{F}=h .
$$

Similarly, we have:

Proposition 5.4. Let $L$ be a zero-dimensional frame and let $h \in \mathrm{C}(L, \mathbb{Z})^{\wedge}$. Then

$$
h=\bigwedge^{\mathrm{IC}(L, \mathbb{Z})}\{g \in \mathrm{C}(L, \mathbb{Z}) \mid h \leq g\} .
$$

Corollary 5.5. Let $L$ be a zero-dimensional frame and let $h \in \mathrm{C}(L, \mathbb{Z})(L)^{\mathrm{W}}$. Then

$$
h=\bigvee^{\mathrm{IC}(L, \mathbb{Z})}\{f \in \operatorname{IC}(L, \mathbb{Z}) \mid f \leq h\}=\bigwedge^{\mathrm{IC}(L, \mathbb{Z})}\{g \in \mathrm{IC}(L, \mathbb{Z}) \mid h \leq g\} .
$$

Now we have the following analogues of Propositions 4.5 and 4.6 in the integervalued case, which can be proved in a similar way.

Proposition 5.6. The class $\mathrm{C}(L, \mathbb{Z})^{\vee}$ is closed under non-void bounded suprema and $\mathrm{C}(L, \mathbb{Z})^{\wedge}$ is closed under non-void bounded infima.

Proposition 5.7. For any zero-dimensional frame $L, \mathrm{C}(L, \mathbb{Z})^{\mathrm{XX}}$ is Dedekind complete.

Corollary 5.8. For any zero-dimensional frame $L, \mathrm{C}(L, \mathbb{Z})^{\mathrm{WX}}$ is the Dedekind completion of $\mathrm{C}(L, \mathbb{Z})$.

Finally, we have a corollary that augments [7, Proposition 3] (the proof goes very similar to that of Corollary 4.8 so we omit it).

Corollary 5.9. For any zero-dimensional frame $L$, the following are equivalent.

(1) L is extremally disconnected.

(2) $\mathrm{C}(L, \mathbb{Z})=\mathrm{C}(L, \mathbb{Z})^{x}$.

(3) $\mathrm{C}(L, \mathbb{Z})$ is Dedekind complete.

(4) $\mathrm{C}(L, \mathbb{Z})$ is closed under non-void bounded suprema. 
It is quite evident now that we could also consider the case of bounded integervalued continuous function. We omit the details.

\section{The classical case}

In this final section we show that the pointfree approach pursued in this paper sheds new light on the classical case of $C(X)$ (for a space $X$ ) and provides a new construction that we believe is more natural than that given by Anguelov in [1]. The construction in [1] works with Hausdorff continuous functions, whereas our construction hinges only on a direct lattice-theoretical approach to the problem.

To begin with, recall from Corollary 2.5 the natural isomorphism

$$
\Phi: \operatorname{IC}(\mathcal{O} X) \rightarrow \mathrm{C}(X, \mathbb{I R})
$$

given, for each $h \in \operatorname{IC}(\mathcal{O} X)$, by

$$
\Phi(h)(x)=[\bigvee\{r \in \mathbb{Q} \mid x \in h(r,-)\}, \bigwedge\{r \in \mathbb{Q} \mid x \in h(-, r)\}]
$$

for all $x \in X$.

Composing $\Phi(h)$ with projections $\pi_{1}$ and $\pi_{2}$, we get a couple of real-valued functions $\pi_{1} \circ \Phi(h), \pi_{2} \circ \Phi(h): X \rightarrow \mathbb{R}$ such that

(i) $\pi_{1} \circ \Phi(h) \leq \pi_{2} \circ \Phi(h)$,

(ii) $\pi_{1} \circ \Phi(h) \in \operatorname{LSC}(X, \mathbb{R})$,

(iii) $\pi_{2} \circ \Phi(h) \in \operatorname{USC}(X, \mathbb{R})$ (recall Remark $2.1(1)$ ).

Lemma 6.1. Let $f, g \in \operatorname{IC}(\mathcal{O} X)$. Then:

(1) $\pi_{1} \circ \Phi(f) \leq \pi_{1} \circ \Phi(g)$ if and only if $f(r,-) \leq g(r,-)$ for all $r \in \mathbb{Q}$.

(2) $\pi_{2} \circ \Phi(f) \geq \pi_{2} \circ \Phi(g)$ if and only if $f(-, r) \leq g(-, r)$ for all $r \in \mathbb{Q}$.

Proof. To check (1), first consider $f, g \in \operatorname{IC}(\mathcal{O} X)$ such that

$$
\pi_{1} \circ \Phi(f) \leq \pi_{1} \circ \Phi(g)
$$

and let $r \in \mathbb{Q}$. Then, for any $s>r$ in $\mathbb{Q}$ and $x \in f(s,-)$ one has

$$
r<s \leq \bigvee\{p \in \mathbb{Q} \mid x \in f(p,-)\} \leq \bigvee\{p \in \mathbb{Q} \mid x \in g(p,-)\}
$$

and thus there exists a $p>r$ in $\mathbb{Q}$ such that $x \in g(p,-) \leq g(r,-)$. Consequently, $f(r,-)=\bigvee_{s>r} f(s,-) \leq g(r,-)$. The reverse implication is straightforward. 
In order to check (2) note first that $\Phi(-f)=-\Phi(f), \pi_{1}(-f)=-\pi_{2}(f)$ and $\pi_{2}(-f)=-\pi_{1}(f)$. Thus $f(-, r) \leq g(-, r)$ for any $r \in \mathbb{Q}$ if and only if $-f(r,-) \leq-g(r,-)$ for any $r \in \mathbb{Q}$. Then, by statement (1), this is equivalent to $\pi_{1} \circ \Phi(-f) \leq \pi_{1} \circ \Phi(-g)$, that is, $-\left(\pi_{2} \circ \Phi(f)\right) \leq-\left(\pi_{2} \circ \Phi(g)\right)$.

In particular, this implies that $\Phi$ is an order isomorphism for both $\leq$ and $\sqsubseteq$. Furthermore, its restriction to $\mathrm{C}(\mathcal{O} X)$ and $\mathrm{C}(X)$ is also an order isomorphism. Then, using Lemma 6.1, the following facts follow immediately.

Fact 6.2. Let $h \in \operatorname{IC}(\mathcal{O} X)$ and let $f, g \in \mathrm{C}(\mathcal{O} X)$ such that $f \leq h \leq g$. Then:

(1) $h \in \mathrm{C}(\mathcal{O} X)^{\vee}$ if and only if

$$
\Phi(h) \sqsubseteq h^{\prime} \Longrightarrow \pi_{2} \circ \Phi(h)=\pi_{2}\left(h^{\prime}\right) \text { in } \mathrm{C}(X, \mathbb{I} \mathbb{R}) .
$$

(2) $h \in \mathrm{C}(\mathcal{O} X)^{\wedge}$ if and only if

$$
\Phi(h) \sqsubseteq h^{\prime} \Longrightarrow \pi_{1} \circ \Phi(h)=\pi_{1}\left(h^{\prime}\right) \text { in } \mathrm{C}(X, \mathbb{I} \mathbb{R}) .
$$

(3) $h \in \mathrm{C}(\mathcal{O} X)^{\mathbb{X}}$ if and only if

$$
\Phi(h) \sqsubseteq h^{\prime} \Longrightarrow \Phi(h)=h^{\prime} \text { in } \mathrm{C}(X, \mathbb{R} \mathbb{R}) .
$$

This ensures that $\Phi$ yields order isomorphisms between $\mathrm{C}(\mathcal{O} X)^{\vee}, \mathrm{C}(\mathcal{O} X)^{\wedge}$ and $\mathrm{C}(\mathcal{O} X)^{\mathrm{W}}$ (ordered by $\leq$ ), respectively, and classes

$$
\begin{gathered}
\mathrm{C}(X)^{\vee}=\{h \in \mathrm{C}(X, \mathbb{I R}) \mid \text { (a) there exist } f, g \in \mathrm{C}(X) \text { such that } f \leq h \leq g, \\
\text { (b) } \left.h \sqsubseteq h^{\prime} \Longrightarrow \pi_{2}(h)=\pi_{2}\left(h^{\prime}\right)\right\}, \\
\mathrm{C}(X)^{\wedge}=\{h \in \mathrm{C}(X, \mathbb{I}) \mid \text { (a) there exist } f, g \in \mathrm{C}(X) \text { such that } f \leq h \leq g, \\
\mathrm{C}(X)^{x}=\{h \in \mathrm{C}(X, \mathbb{I} \mathbb{R}) \mid \text { (a) there exist } f, g \in \mathrm{C}(X) \text { such that } f \leq h \leq g, \\
\text { (b) } \left.h \sqsubseteq h^{\prime} \Longrightarrow h=h^{\prime}\right\} .
\end{gathered}
$$

Additionally, notice that $h \in \operatorname{IC}(\mathcal{O} X)$ is constant if and only if $\Phi(h)$ is constant in $\mathrm{C}(X, \mathbb{I R})$ and that $h \in \operatorname{IC}(\mathcal{O} X)$ is $\mathbb{Z}$-valued if and only if both $\pi_{1} \circ \Phi(h)$ and $\pi_{2} \circ \Phi(h)$ take values in $\mathbb{Z}$. 
For the sake of completeness, let us also introduce the following classes:

$$
\begin{aligned}
& \mathrm{C}^{*}(X)^{\vee}=\left\{h \in \mathrm{C}(X)^{\vee} \mid \exists p, q \in \mathbb{Q} \text { such that } h(x) \subseteq[p, q] \text { for all } x \in X\right\}, \\
& \mathrm{C}^{*}(X)^{\wedge}=\left\{h \in \mathrm{C}(X)^{\vee} \mid \exists p, q \in \mathbb{Q} \text { such that } h(x) \subseteq[p, q] \text { for all } x \in X\right\} \text {, } \\
& \mathrm{C}^{*}(X)^{\mathfrak{X}}=\left\{h \in \mathrm{C}(X)^{\mathfrak{X}} \mid \exists p, q \in \mathbb{Q} \text { such that } h(x) \subseteq[p, q] \text { for all } x \in X\right\}, \\
& \mathrm{C}(X, \mathbb{Z})^{\vee}=\left\{h \in \mathrm{C}(X)^{\vee} \mid \pi_{1}(h(x)), \pi_{2}(h(x)) \in \mathbb{Z} \text { for all } x \in X\right\}, \\
& \mathrm{C}(X, \mathbb{Z})^{\wedge}=\left\{h \in \mathrm{C}(X)^{\vee} \mid \pi_{1}(h(x)), \pi_{2}(h(x)) \in \mathbb{Z} \text { for all } x \in X\right\}, \\
& \mathrm{C}(X, \mathbb{Z})^{\mathfrak{W}}=\left\{h \in \mathrm{C}(X)^{\mathfrak{X}} \mid \pi_{1}(h(x)), \pi_{2}(h(x)) \in \mathbb{Z} \text { for all } x \in X Z\right\} .
\end{aligned}
$$

Analogously, they are order isomorphic to $\mathrm{C}^{*}(\mathcal{O} X)^{\vee}, \mathrm{C}^{*}(\mathcal{O} X)^{\wedge}, \mathrm{C}^{*}(\mathcal{O} X)^{\mathrm{W}}$, $\mathrm{C}(\mathcal{O} X, \mathbb{Z})^{\vee}, \mathrm{C}(\mathcal{O} X, \mathbb{Z})^{\wedge}$ and $\mathrm{C}(\mathcal{O} X, \mathbb{Z})^{\text {Wx }}$ (ordered by $\leq$ ), respectively.

Finally, recall that $\mathcal{O} X$ is completely regular (resp. extremally disconnected, zero-dimensional) as a frame if and only if the space $X$ is completely regular (resp. extremally disconnected, zero-dimensional). Then, from Corollaries 3.7, 4.7, 4.8, 5.8 and 5.9 it follows immediately that:

Proposition 6.3. For any completely regular topological space $(X, \mathcal{O} X)$,

(1) $\mathrm{C}(X)^{\mathrm{W}}$ is the Dedekind completion of $\mathrm{C}(X)$.

(2) $\mathrm{C}^{*}(X)^{\mathrm{W}}$ is the Dedekind completion of $\mathrm{C}^{*}(X)$.

Corollary 6.4. For any completely regular topological space $(X, \mathcal{O} X)$, the following are equivalent.

(1) $X$ is extremally disconnected.

(2) $\mathrm{C}(X)=\mathrm{C}(X)^{\mathrm{XX}}$.

(3) $\mathrm{C}(X)$ is Dedekind complete.

(4) $\mathrm{C}(X)$ is closed under non-void bounded suprema.

(5) $\mathrm{C}^{*}(X)=\mathrm{C}^{*}(X)^{\mathrm{XX}}$.

(6) $\mathrm{C}^{*}(X)$ is Dedekind complete.

(7) $\mathrm{C}^{*}(X)$ is closed under non-void bounded suprema.

Proposition 6.5. For any zero-dimensional topological space $(X, \mathcal{O} X), \mathrm{C}(X, \mathbb{Z})^{\mathrm{XX}}$ is the Dedekind completion of $\mathrm{C}(X, \mathbb{Z})$. 
Corollary 6.6. For any zero-dimensional topological space $(X, \mathcal{O} X)$, the following are equivalent.

(1) $X$ is extremally disconnected.

(2) $\mathrm{C}(X, \mathbb{Z})=\mathrm{C}(X, \mathbb{Z})^{\mathrm{W}}$.

(3) $\mathrm{C}(X, \mathbb{Z})$ is Dedekind complete.

(4) $\mathrm{C}(X, \mathbb{Z})$ is closed under non-void bounded suprema.

We close with a comment regarding the relation of our results above to the construction of Anguelov [1]. For that we need to recall the well-known fact that each real-valued function $f: X \rightarrow \mathbb{R}$ on a space $X$ admits an upper regularization $f^{-} \in \operatorname{USC}(X, \overline{\mathbb{R}})$, where $\overline{\mathbb{R}}=\mathbb{R} \cup\{-\infty,+\infty\}$, defined by

$$
f^{-}(x)=\bigwedge\{\bigvee f(U) \mid x \in U \in \mathcal{O} X\} \quad \text { for all } x \in X
$$

This is the smallest upper semicontinuous majorant of $f$, i.e.,

$$
f^{-}=\bigwedge\{g \in \operatorname{USC}(X, \overline{\mathbb{R}}) \mid f \leq g\} .
$$

Dually, $f$ admits a lower regularization $f^{\circ} \in \operatorname{LSC}(X, \overline{\mathbb{R}})$ defined by

$$
f^{\circ}(x)=\bigvee\{\bigwedge f(U) \mid x \in U \in \mathcal{O} X\} \quad \text { for all } x \in X,
$$

and $f^{\circ}$ is the biggest lower semicontinuous minorant of $f$, i.e.,

$$
f^{\circ}=\bigvee\{g \in \operatorname{LSC}(X, \overline{\mathbb{R}}) \mid g \leq f\} .
$$

It is then not hard to check that

$$
\begin{gathered}
\mathrm{C}(X)^{\vee}=\left\{h \in \mathrm{C}(X, \mathbb{R}) \mid \exists f, g \in \mathrm{C}(X): f \leq h \leq g \text { and } \pi_{1}(h)^{-}=\pi_{2}(h)\right\}, \\
\mathrm{C}(X)^{\wedge}=\left\{h \in \mathrm{C}(X, \mathbb{R}) \mid \exists f, g \in \mathrm{C}(X): f \leq h \leq g \text { and } \pi_{2}(h)=\pi_{1}(h)^{\circ}\right\}, \\
\mathrm{C}(X)^{\aleph \times}=\left\{h \in \mathrm{C}(X, \mathbb{R}) \mid \exists f, g \in \mathrm{C}(X): f \leq h \leq g, \pi_{1}(h)=\pi_{1}(h)^{\circ}\right. \\
\text { and } \left.\pi_{1}(h)=\pi_{1}(h)^{\circ}\right\} .
\end{gathered}
$$

For instance, for the first, given $h \in \mathrm{IC}(X)$ and $f, g \in \mathrm{C}(X)$ such that $f \leq h \leq g$ and $\pi_{2}(h)=\pi_{2}(j)$ whenever $h \sqsubseteq j$, since $h \sqsubseteq\left[\pi_{1}(h), \pi_{1}(h)^{-}\right]$, it follows that $\pi_{2}(h)=\pi_{1}(h)^{-}$. Conversely, let $h \in \operatorname{IC}(X)$ be such that $\pi_{2}(h)=\pi_{1}(h)^{-}$and $h \sqsubseteq j$, i.e., $\pi_{1}(h) \leq \pi_{1}(j) \leq \pi_{2}(j) \leq \pi_{2}(h)$. Then

$$
\pi_{2}(h)=\pi_{1}(h)^{-} \leq \pi_{1}(j)^{-} \leq \pi_{2}(j) \leq \pi_{2}(h)
$$

and so $\pi_{2}(h)=\pi_{2}(j)$. The other identities follow similarly.

This description of the Dedekind completion of $\mathrm{C}(X)$ is precisely the one given by the construction of Anguelov in terms of Hausdorff continuous functions presented in [1]. 


\section{Bibliography}

[1] R. Anguelov, Dedekind completion of C (X) by Hausdorff continuous functions, Quaest. Math. 27 (2004), no. 2, 153-169.

[2] R. N. Ball, Convergence and Cauchy structures on lattice ordered groups, Trans. Amer. Math. Soc. 259 (1980), 357-392.

[3] B. Banaschewski, The real numbers in pointfree topology, Textos Mat. Sér. B, vol. 12, Departamento de Matemática da Universidade de Coimbra, 1997.

[4] B. Banaschewski, A uniform view of localic realcompactness, J. Pure Appl. Algebra 143 (1999) 49-68.

[5] B. Banaschewski and G. Bruns, Categorical characterization of the MacNeille completion, Arch. Math. 18 (1967), 369-377.

[6] B. Banaschewski, J. Gutiérrez García and J. Picado, Extended real functions in pointfree topology, J. Pure Appl. Algebra 216 (2012), 905-922.

[7] B. Banaschewski and S. Hong, Completeness properties of function rings in pointfree topology, Comment. Math. Univ. Carolin. 44 (2003), 245-259.

[8] G. Birkhoff, Lattice Theory, Amer. Math. Soc. Coll. Publ. 25, 3rd ed., Providence, R.I., 1979.

[9] A. Bishop, A universal mapping characterization of the completion by cuts, Algebra Universalis 8 (1978), 349-353.

[10] P. Conrad and D. McAlister, The completion of a lattice ordered group, J. Austral. Math. Soc. 9 (1969), 182-208.

[11] N. Dăneţ, The Dedekind completion of $C(X)$ : An interval-valued functions approach, Quaest. Math. 34 (2011), 213-223.

[12] G. Gierz, K. H. Hofmann, K Keimel, J. D. Lawson, M. Mislove and D. S. Scott, Continuous Lattices and Domains, Encyclopedia of Mathematics and its Applications, vol. 93, Cambridge University Press, Cambridge, 2003.

[13] J. Gutiérrez García and J. Picado, Rings of real functions in pointfree topology, Topology Appl. 158 (2011), 2264-2278.

[14] P. T. Johnstone, Stone Spaces, Cambridge Studies in Advanced Mathematics, vol. 3, Cambridge University Press, Cambridge, 1982.

[15] D. G. Johnson, The completion of an archimedean f-ring, J. London Math. Soc. 40 (1965), 493-496.

[16] J. E. Mack and D. G. Johnson, The Dedekind completion of $C(\mathcal{X})$, Pacific J. Math. 20 (1967), 231-243.

[17] H. MacNeille, Partially ordered sets, Trans. Amer. Math. Soc. 42 (1937), 416-460. 
[18] H. Nakano, Über das System aller stetigen Funktionen auf einen topologischen Raum, Proc. Imp. Acad. Tokyo 17 (1941), 308-310.

[19] J. Picado and A. Pultr, Frames and locales: Topology without points, Frontiers in Mathematics, vol. 28, Springer, Basel, 2012.

[20] J. Schmidt, Each join-completion of a partially ordered set is the solution of a universal problem, J. Austral. Math. Soc. 17 (1974), 406-413.

[21] D. S. Scott, Lattice theory, data types and semantics, in: R. Rustin (editor), Formal semantics of programming languages, vol. 2 of Courant Computer Science Symposis, pp. 65-106, Prentice-Hall, 1972.

[22] S. A. Steinberg, Lattice-ordered rings and modules, Springer, New York, 2010.

[23] M. H. Stone, Boundedness properties in function lattices, Canad. J. Math. 1 (1949), 176-186.

\section{Author information}

Imanol Mozo Carollo, Department of Mathematics, University of the Basque Country UPV/EHU, Apdo. 644, 48080 Bilbao, Spain.

E-mail: imanolmozo@gmail.com

Javier Gutiérrez García, Department of Mathematics, University of the Basque Country UPV/EHU, Apdo. 644, 48080 Bilbao, Spain.

E-mail: javier.gutierrezgarcia@ehu.es

Jorge Picado, CMUC, Department of Mathematics, University of Coimbra, 3001-501 Coimbra, Portugal. E-mail: picado@mat.uc.pt 